\title{
Programación de un Sistema de Fabricación tipo "Job Shop" bajo un Enfoque de Sostenibilidad
}

\author{
Germán A. Coca ${ }^{(1)}$, Omar D. Castrillón ${ }^{(2)}$ y Santiago Ruiz-Herrera ${ }^{(2)}$ \\ (1) Universidad Escuela de Ingeniería de Antioquia. Alto de las Palmas. Medellín - Colombia. \\ (e-mail: german.coca@eia.edu.co) \\ (2) Universidad Nacional de Colombia, Sede Manizales, Facultad de Ingeniería y Arquitectura, \\ Departamento de Ingeniería Industrial, Campus la Nubia, CP 170001, Manizales - Colombia. \\ (e-mail: odcastrillong@unal.edu.co; sruizhe@unal.edu.co)
}

Recibido Abr. 19, 2016; Aceptado Jun. 29, 2016; Versión final Jul. 29, 2016, Publicado Dic. 2016

\begin{abstract}
Resumen
En este artículo se establece un enfoque novedoso para programar sistemas Job Shop, basado en sostenibilidad. Se evalúan las siguientes variables de sostenibilidad: emisiones equivalentes de dióxido de carbono (variable ambiental), intensidad sonora, accidentalidad laboral y peso manipulado (variables sociales: salud ocupacional) y finalmente, tiempo de proceso y costos de mano de obra directa (variables económicas). La metodología se estructura en catorce pasos que cubren aspectos tales como diseño del método multiobjetivo, fundamentos de los algoritmos genéticos y comparación con otros métodos. Se determina que el método diseñado muestra un desempeño superior para la variable factor ponderado $\left(\mathrm{P}\left(\mathrm{X}_{\mathrm{j}}\right)\right.$, al compararlo con resultados de otros métodos tales como reglas de programación o el método del cuello de botella móvil.
\end{abstract}

\section{Programming in a Manufacturing System of the "Job Shop" type with a Focus on Sustainability}

\begin{abstract}
In this paper an innovative focus to programming Job Shop systems, based on sustainability, is presented. The following sustainability variables are evaluated: carbon dioxide emissions (environmental variable), sound intensity, accident rate and handled weight (social variables: occupational health) and finally, makespan time and direct labor costs (economic variables). The methodology is structured in fourteen steps that cover aspects such as multiobjective method design, description of genetic algorithms, and comparison with other methods. It is determined that the designed method shows greater performance for the variable weighted factor $\mathrm{P}(\mathrm{Xj})$ when they are compared with results from other methods such as programming rules and mobile bottle neck method.
\end{abstract}

Keywords: sustainability; carbon dioxide; accident rate; Job Shop; genetic algorithms 


\section{INTRODUCCIÓN}

En la actualidad, debido a las tendencias de desarrollo internacional, se hace necesario gestionar las organizaciones, bajo un enfoque fundamentado en los conceptos de sostenibilidad (Pagell y Shevchenko, 2014). De esta forma, se señala que en la literatura de corriente principal, el sistema de manufactura "Job Shop" se ha investigado, buscando incrementar la eficiencia de las diversas operaciones intervinientes en el sistema productivo (Shen et al., 2014). De manera consecuente, se plantea que en la programación de tal clase de sistema de manufactura, se ha desconocido la importancia de minimizar los impactos negativos, ya sea respecto a la salud de la persona o respecto al medio ambiente (Becic et al., 2012). Es así como, en este artículo se evalúa el sistema de operaciones de una compañía del sector metalmecánico, clasificada bajo el tipo: "Job Shop". Para la organización objeto de estudio, se ilustra a continuación el comportamiento de las variables de sostenibilidad: tiempo de proceso y costos de mano de obra directa (variables económicas), emisiones equivalentes de dióxido de carbono (variable ambiental), y por último: intensidad sonora, accidentalidad laboral y peso manipulado en promedio por turno de trabajo (variables sociales: salud en el trabajo).

En el caso de la variable, tiempo de proceso, se afirma que la empresa analizada, utiliza reglas de programación, con el propósito de establecer, la categoría de tiempo en cuestión. Al respecto, se menciona que la aplicación de esta clase de reglas, incide representativamente, en la obtención de bajos niveles de productividad (López et al., 2015). A su vez, en referencia a los costos de mano de obra directa, se observa como la demanda fluctuante de la compañía, genera dificultades para definir, aquel esquema de trabajo, asociado con el mínimo costo de operación. Igualmente, desde la dirección de la organización, se ha determinado que las emisiones equivalentes de dióxido de carbono, constituyen su mayor riesgo ambiental. En este sentido, se ha decidido implementar estrategias de producción que permitan reducir dicho nivel de emisiones. De otro lado, para la situación particular relacionada con la accidentalidad laboral, el área de salud ocupacional, ha identificado que en promedio, cada cuatro días suceden accidentes de trabajo, los cuales requieren incapacidad médica en el $60 \%$ de los casos. De este modo, la administradora de riesgos laborales, ha detectado que dicho nivel de accidentalidad ocurre en gran parte, como consecuencia de la manipulación de objetos pesados en los diversos puestos de trabajo. Finalmente, en cuanto a la intensidad sonora, se aprecian los siguientes hechos: $100 \%$ de las personas, se encuentran expuestas a intensidades sonoras no permisibles y $40 \%$ de los colaboradores del área productiva, presentan hipoacusia laboral.

Al tomar referencia en lo argumentado, se indica que el presente artículo tiene como propósito minimizar de forma simultánea el comportamiento de las variables: tiempo de proceso, costos de mano de obra directa, emisiones equivalentes de dióxido de carbono, nivel de intensidad sonora, accidentalidad laboral y peso promedio manipulado por turno de trabajo. Para éste efecto, se estructura un nuevo algoritmo híbrido, el cual fusiona los siguientes conceptos: carga por centro de trabajo, procedimiento estándar de algoritmos genéticos multiobjetivo, análisis de poblaciones y búsqueda local iterada. Complementariamente, se denota que el análisis simultáneo de las variables consideradas se ha abordado de manera superficial en los procesos de investigación y asimismo, se enuncia que el marco teórico correspondiente a la salud ocupacional, ha sido poco explorado en la programación de operaciones de los sistemas Job Shop (Shen et al., 2014).

Con relación al tratamiento de las variables de interés, se establece la necesidad de determinar en primer término, el tiempo óptimo o sub-óptimo de proceso. Es así como, la definición de la última variable, dada la existencia de un número "P" pedidos y de un número "Q" centros de trabajo, requiere la evaluación de $P ! Q$ puntos posibles de solución (Castrillón et al., 2011; Karimi et al., 2015; Nicoara, 2012). De forma consecuente, ante la imposibilidad de explorar P!Q soluciones, utilizando la fundamentación aplicable a la investigación de operaciones (Shen y Yao, 2015), se decide utilizar en este artículo aquella técnica denominada, algoritmos genéticos. Respecto a los algoritmos genéticos, se identifican dos categorías generales de estudio: diseño de algoritmos en problemas mono-objetivo y problemas multiobjetivo en Job Shop.

Diseño de algoritmos en problemas mono-objetivo. Se aprecian en este caso tres desarrollos. El primero de estos desarrollos, asegura el mejoramiento de los individuos en las nuevas poblaciones, al modificar los operadores de cruzamiento y de mutación (Huang et al., 2014). En el segundo desarrollo, se muestra una metodología que permite incluir de forma ágil nuevos puestos de trabajo, de acuerdo con los cambios en la demanda, en las necesidades de transformación de los materiales y en las especificaciones de los productos (Fang y Yue, 2015). Y en cuanto al tercer desarrollo, se analiza el efecto de la habilidad de los operadores sobre el desempeño del tiempo de procesamiento óptimo o sub-óptimo (Mencía et al., 2014).

Problemas multiobjetivo en "Job Shop". A la categoría pertenecen once desarrollos. Estos once desarrollos, se clasifican en métodos exactos o heurísticos. Desde el enfoque de los métodos exactos, se identificó un 
desarrollo particular, el cual analiza la programación multiobjetivo en “Job Shop”, con el propósito de minimizar variables tales como: tardanza, carga de trabajo, tiempo de flujo, trabajo en proceso y costo de preparación. De esta manera, se implementan para la solución del problema, el siguiente tipo de conceptos: redes de Petri, sistemas de espera, modelos de decisión, cadenas de markov, simulación Montecarlo (Nicoara, 2012).

La aplicación de métodos exactos se complementa con la aplicación de métodos heurísticos. Con base en lo afirmado, se expresa que los otros diez desarrollos clasifican en el campo de la heurística. De forma general, dos de estos desarrollos se fundamentan en el diseño de los algoritmos genéticos multiobjetivo. Asimismo, los siguientes siete desarrollos se estructuran para dar respuesta a cierta necesidad genérica, cuyo análisis se aborda desde la aplicación de métodos híbridos y, por último, el décimo desarrollo, explica las perspectivas del problema multiobjetivo en los sistemas Job Shop.

Al evaluar las dos aplicaciones fundamentadas en el diseño de los algoritmos multipropósito, se establece que su objetivo se orienta hacia la evaluación de algunas variables no tratadas hasta el momento en la literatura. Es así como, en la primera aplicación se implementa NSGA-II, con el fin de minimizar el tiempo de proceso, los costos de manufactura y el consumo de energía (Jiang et al., 2014); mientras que en la segunda aplicación, se propone un algoritmo capaz de superar el rendimiento del algoritmo NSGA-II (Ghiasi et al., 2012), en referencia al comportamiento de las variables: resistencia a la tracción, masa y tiempo de manufactura, durante aquel proceso de fabricación de partes elaboradas con base en fibra de carbono.

Los siguientes siete desarrollos, corresponden a un conjunto de algoritmos híbridos, bajo los cuales se pretende minimizar el comportamiento de las variables: tiempo de proceso, total de carga de trabajo y máxima carga de trabajo (Davarzani y Akbarzadeh-T, 2014; Nagamani et al., 2013; Rudy y Zelasny, 2015; Xiong et al., 2012; Xue et al., 2014; Yang y Gu, 2014; Yuan y Xu, 2013). Con relación a lo anterior, se anota que los autores de los artículos de interés, coinciden en la necesidad de comparar, los resultados establecidos en sus actividades de investigación, respecto a tres problemas registrados en la literatura. De este modo, se señala que los desarrollos en cuestión, evidencian el cumplimiento de aquellas especificaciones de desempeño, presentadas enseguida: velocidad superior de convergencia y mayor diversidad a nivel poblacional.

Finalmente, el décimo desarrollo sintetiza la evolución histórica de los sistemas Job Shop y, a la vez caracteriza, el análisis prospectivo de sus tendencias (Shen et al., 2014). Al respecto, se afirma que los tres criterios utilizados, con el objetivo de programar las operaciones en sistemas "Job Shop", son: eficiencia (determinación de tiempos de desempeño, definición de niveles de inventarios, detección de productos no conformes), robustez (representación integral de variables de los sistemas Job Shop) y estabilidad (evaluación del comportamiento estocástico de los datos). Por otra parte, en cuanto a las variables de sostenibilidad social y ambiental objeto de este artículo, se determinan en la literatura, los siguientes aspectos:

La intensidad sonora y el peso manipulado en el puesto de trabajo (variables: salud en el trabajo), no se han evaluado de manera profunda como tampoco de manera preliminar, en sus relaciones con las variables intervinientes, en aquel proceso correspondiente, a la programación de producción de los sistemas Job Shop. La accidentalidad laboral (variable asociada con la salud en el trabajo), sólo se ha evaluado en un artículo. Respecto a este desarrollo (Ruiz et al., 2012), se determina que su alcance está limitado a tomar como referente, en el proceso de obtención de los planes detallados de producción, una curva de probabilidad horaria correspondiente a la accidentalidad laboral, la cual se estimó mediante un ajuste de la tasa de accidentalidad reportada en 1999, para el sector metalmecánico en Colombia. Adicionalmente, se establece que en el desarrollo académico revisado, no se identifican las variables intervinientes en la accidentalidad laboral, como tampoco se evalúa la factibilidad de su incorporación en el modelo multiobjetivo.

Al evaluar aquellos artículos relacionados con el consumo de energía, en el campo de los sistemas Job Shop, se detectan seis desarrollos. De este modo, en el primer desarrollo se propone una modificación a la estructura operativa del algoritmo NSGA-II, de tal forma que puedan obtenerse poblaciones caracterizadas por su diversidad, en el respectivo proceso de optimización de las siguientes variables: tiempo proceso, costos producción y consumo energía. (Jiang et al., 2014). En cuanto al segundo desarrollo, se observa la utilización del algoritmo NSGA-II, en aquel proceso correspondiente a la minimización del comportamiento de los parámetros: consumo de energía (preparación de máquina, detención de máquina al finalizar la labor, tiempos de espera) y asimismo, tardanza total (Liu et al., 2014). En el tercer desarrollo, se busca minimizar el comportamiento de las variables: consumo de energía (preparación; detención; tiempos de espera; adaptación a procesos de suspensión programada del servicio de energía, por parte de un organismo regulador), costos de producción y tardanza total, a través de la implementación del algoritmo NSGA-II (Liu 
et al., 2015). En referencia al cuarto desarrollo, se aprecia la minimización del costo total de producción, incluyendo el costo de energía inherente a la operación de aquellos equipos asignados, al sistema de manufactura (Moon y Park, 2014). En el quinto desarrollo, se aplica el algoritmo NSGA-II, con el propósito de minimizar el comportamiento de los parámetros: tiempo de proceso y consumo de energía por equipo (tiempos de preparación; tiempos de espera; determinación de secuencias de cambio de herramientas de corte, según el tipo de procesamiento requerido) (May et al., 2015). Finalmente, en el sexto desarrollo se ilustra tanto la minimización como el pronóstico del consumo de energía en un sistema Job Shop automatizado, utilizando para el caso, aquel software de optimización denominado ILOG OPL (Raileanu et al.,2015).

Respecto a los artículos presentados en el párrafo anterior, se argumenta en términos generales que realizan su énfasis en los aspectos asociados con el consumo de energía eléctrica por cada equipo de procesamiento de materiales, sin explorar con profundidad el consumo de energía, proveniente de otros equipos de producción que utilicen para su funcionamiento combustibles de origen fósil o de otros equipos de apoyo al sistema de producción (sistemas de manejo de materiales, sistemas de iluminación adaptados a los requerimientos de los puestos de trabajo, equipos de mantenimiento, equipos de laboratorio, equipos para desarrollo de nuevos productos, entre otros) que requieran para su operación, ya sea energía eléctrica o energía generada por el uso de combustibles de origen fósil.

Por último, en cuanto a los 6 desarrollos detectados en el tema de sostenibilidad, para sistemas Job Shop, se determina también que el método aquí propuesto, supera a los otros métodos, en el número de variables analizadas, así: $100 \%$, en $50 \%$ de los casos (3 de 6 métodos); $200 \%$ en $50 \%$ de los casos y $140 \%$, en relación al número promedio de variables evaluadas, por los 6 métodos en su conjunto (ver tabla 14). A partir de lo ilustrado en los párrafos previos, puede aseverarse que la conceptualización definida, alrededor de la sostenibilidad, en la programación de los sistemas Job Shop, ha sido parcialmente explorada desde el campo académico; por tanto, se concluye que existen oportunidades de desarrollo investigativo al respecto.

\section{MATERIALES Y MÉTODOS}

En la presente metodología, se propone un método a través del cual se busca programar los sistemas Job Shop, bajo un enfoque de sostenibilidad. Con relación a lo anterior, se expresa que el método en cuestión fusiona algunos elementos provenientes de los conceptos: carga de trabajo, algoritmos genéticos y búsqueda local. De esta manera, se procede a desarrollar en primera instancia la síntesis de la fase correspondiente a la calibración del algoritmo; luego, se aprecia el pseudocódigo del mismo algoritmo y por último, se observa la descripción por pasos del método propuesto. En consecuencia con lo previamente señalado, en los pasos 1 a 9 se establece la caracterización del método diseñado; más adelante, en el paso 10 se describen los elementos fundamentales de la revisión sistemática de literatura, en el campo específico de la programación de los sistemas Job Shop, bajo un enfoque de sostenibilidad; posteriormente, en los pasos 11 a 13 se compara aquel valor de "factor ponderado $\left(P\left(X_{j}\right)\right)$ " que se obtuvo, después de ejecutar este método, respecto a aquellos valores de "factor ponderado $\left(P\left(X_{j}\right)\right)$ " determinados, tras aplicar diferentes métodos (esquema de organización de la compañía objeto de estudio, reglas de programación y cuello de botella móvil). Finalmente, en el paso 14, se explica el análisis de varianza de los resultados arrojados por el método propuesto.

De acuerdo con lo indicado en el párrafo anterior, la calibración del algoritmo, se efectuó a partir de la evaluación de aquellos puntos de probabilidad que son utilizados habitualmente, en el desarrollo de algoritmos de carácter genético: 0.01 a 0.06 en el caso del operador de mutación y a su vez, 0.9 a 1 en el caso del operador de cruzamiento (ver puntos de entrada en figura 1). De esta forma, se ejecutaron los procesos de análisis necesarios, con el propósito de determinar los parámetros de los operadores, por medio de los cuales podrían obtenerse valores máximos, en el comportamiento de la variable de medición general: factor ponderado (ver etapa 1 de la figura 1 y el concepto de factor ponderado en el paso № 9 de la metodología). Al respecto, se detectó que se logra alcanzar desempeño superior en el comportamiento de la última variable, para aquel conjunto de combinaciones de probabilidad, cuyos valores presentan simultáneamente las siguientes características: puntos de mutación mayores o iguales a $5 \%$ y asimismo, puntos de cruzamiento mayores o iguales a $97 \%$ (ver puntos proceso en figura 1). Más adelante, para las combinaciones de probabilidad que inciden en el comportamiento óptimo o sub-óptimo de la variable: factor ponderado, se procedió a identificar la combinación que minimiza el comportamiento de la variable: tiempo de cómputo (ver etapa 2 en figura 1). Con relación a lo descrito, se encontró que las probabilidades de mutación y de cruzamiento, cuyos valores ascienden respectivamente a: $5 \%$ y $97 \%$ (ver selección en figura 1), generan los menores tiempos de proceso ( 340.5 segundos promedio para 30 ejecuciones del programa). En consecuencia, se expone que los niveles de probabilidad establecidos, constituyen los parámetros de operación (mutación: 5 \% y cruzamiento: $97 \%$ ), a ser implementados en el diseño del presente algoritmo. Igualmente, en la figura 2 se presenta el pseudocódigo del algoritmo: 


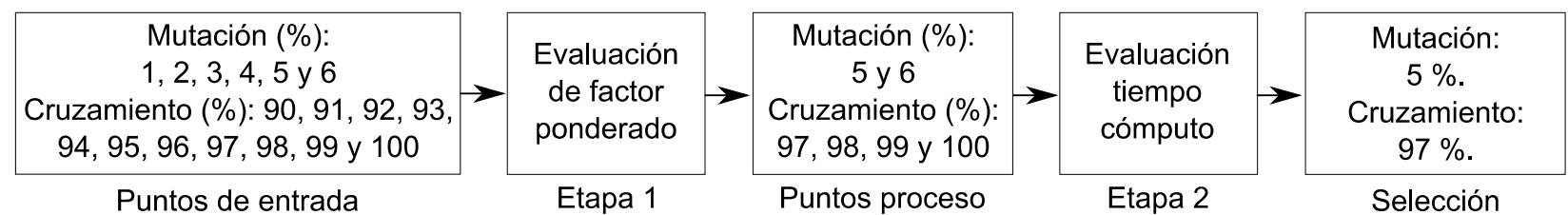

Fig. 1: Procedimiento general de calibración del algoritmo.

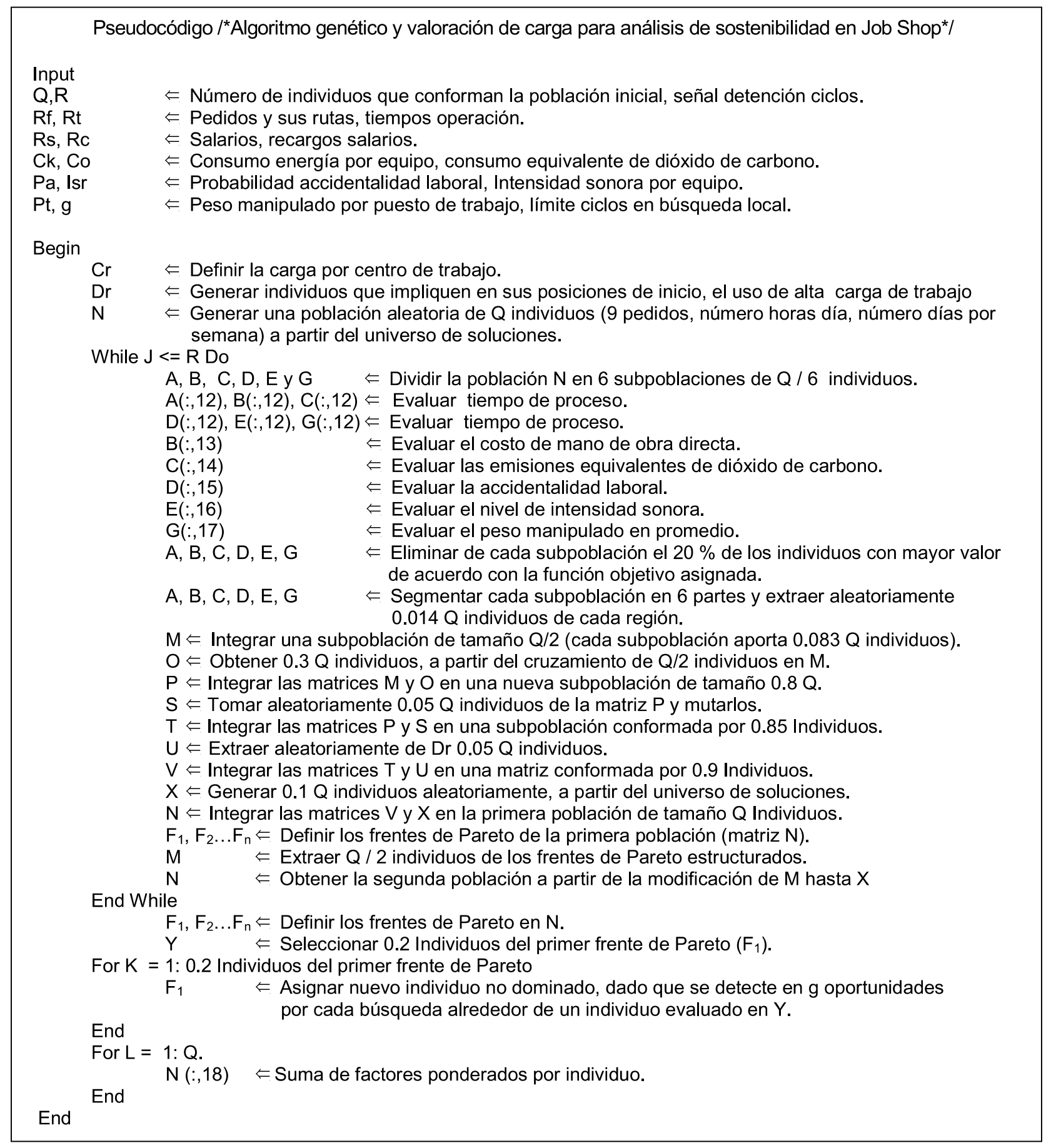

Fig. 2: Pseudocódigo del algoritmo.

\section{Paso 1. Descripción del individuo a ser evaluado}

De acuerdo con las condiciones de la compañía analizada, existen 9 pedidos a ser programados. Estos pedidos después de permutarse equivalen a 9! individuos. Cada individuo contiene 6 subindividuos. Un subindividuo, se describe por medio de las columnas de la tabla 1: "Individuo", "turnos de labor" y "trabaja con o sin dominical". Asimismo, en la columna "Individuo", se aprecia como ejemplo el individuo: "2-4-9-7-1- 
8-3-6-5", el cual proviene de una de las 9! permutaciones ya nombradas. En este individuo, se interpreta que el "pedido 2" sería el primer pedido en procesarse, mientras que el "pedido 5" sería el último pedido en ingresar a fabricación.

Para cada individuo, se establecen diferentes esquemas de organización por turno. Estos esquemas se ilustran en las columnas de la tabla 1: "Turnos de labor" al igual que "horas día". Con base en la información de estas columnas, se determina que un individuo puede proyectarse en tres horizontes de tiempo: día de 8 horas (6 a.m a 2 p.m), día de 16 horas (6 a.m a 2 p.m y 2 p.m a 10 p.m) o día de 24 horas (6 a.m a 2 p.m, 2 p.m a 10 p.m y 10 p.m a 6 a.m). A su vez, en la columna: "trabaja con o sin dominical", se indica que el individuo también puede evaluarse en dos alternativas de tiempo a la semana: 7 días (con dominical) o seis días (sin dominical). Enseguida se muestra la tabla 1.

Tabla 1: Esquemas de organización por turno.

\begin{tabular}{|c|c|c|c|c|}
\hline $\begin{array}{c}\text { Número } \\
\text { subindividuo }\end{array}$ & Individuo & Turnos de labor & $\begin{array}{l}\text { Horas } \\
\text { Día }\end{array}$ & $\begin{array}{l}\text { Trabaja con o } \\
\text { sin dominical }\end{array}$ \\
\hline 1 & $2-4-9-7-1-8-3-6-5$ & 6 am a 2 pm & 8 & Con dominical \\
\hline 2 & $2-4-9-7-1-8-3-6-5$ & 6 am a $2 p m-2 p m$ a $10 p m$ & 16 & Con dominical \\
\hline 3 & $2-4-9-7-1-8-3-6-5$ & 6 am a $2 p m-2 p m$ a $10 p m-10 p m$ a 6 am & 24 & Con dominical \\
\hline 4 & $2-4-9-7-1-8-3-6-5$ & 6 am a 2 pm & 8 & Sin dominical \\
\hline 5 & $2-4-9-7-1-8-3-6-5$ & 6 am a $2 p m-2 p m$ a $10 p m$ & 16 & Sin dominical \\
\hline 6 & $2-4-9-7-1-8-3-6-5$ & 6 am a $2 p m-2 p m$ a $10 p m-10 p m$ a 6 am & 24 & Sin dominical \\
\hline
\end{tabular}

A cada subindividuo de la tabla 1, se le denominará en adelante individuo. Es así como, la estructura de un individuo al igual que su respectivo ejemplo, se muestran en la tabla 2 . En esta estructura se aprecian 11 celdas, de las cuales se utilizan nueve, para almacenar cada uno de los pedidos. Por ejemplo, en la tabla en mención se establece que a la "celda 1" se le relaciona el "pedido 2", significando que este pedido sería el primero en programarse. Al incrementar el número de la celda y avanzar hasta la "celda 9", se identifica que a esta celda le corresponde el "pedido 5", determinándose para la situación particular que el pedido considerado, debe programarse en novena posición. En cuanto a la "celda 10", se registran los "Turnos de labor", es decir, el número de horas laboradas al día (para el ejemplo mostrado se laborarían 8 horas en el día: inicia a las $2 \mathrm{pm}$ y finaliza a las $10 \mathrm{pm}$ ). Finalmente, la "celda 11" hace referencia a trabajar o no el día domingo, es decir, en la "celda11" se indica el número de días laborados a la semana (para el ejemplo diseñado, se laborarían 7 días a la semana, debido a que se encuentra registrada la expresión: "con dominical").

Tabla 2: Estructura y ejemplo de un individuo.

\begin{tabular}{|c|c|c|c|c|c|c|c|c|c|c|}
\hline $\begin{array}{c}\text { Celda } \\
1\end{array}$ & $\begin{array}{c}\text { Celda } \\
2\end{array}$ & $\begin{array}{c}\text { Celda } \\
3\end{array}$ & $\begin{array}{c}\text { Celda } \\
4\end{array}$ & $\begin{array}{c}\text { Celda } \\
5\end{array}$ & $\begin{array}{c}\text { Celda } \\
6\end{array}$ & $\begin{array}{c}\text { Celda } \\
7\end{array}$ & $\begin{array}{c}\text { Celda } \\
8\end{array}$ & $\begin{array}{c}\text { Celda } \\
9\end{array}$ & $\begin{array}{c}\text { Celda } 10 \\
\text { Turnos labor }\end{array}$ & $\begin{array}{c}\text { Celda } 11 \\
\text { Labora con o sin } \\
\text { dominical }\end{array}$ \\
\hline Ped 2 & Ped 4 & Ped 9 & Ped 7 & Ped 1 & Ped 8 & Ped 3 & Ped 6 & Ped 5 & 2 pm a 10 pm & Con dominical \\
\hline
\end{tabular}

Paso 2. Determinación y ordenamiento de la carga asignada por centros de trabajo.

El tiempo de carga, se refiere a la sumatoria de los tiempos de proceso en las diferentes operaciones. De esta manera, se determina el nivel de carga por operación o por centro de trabajo. Con posterioridad, se ordenan las operaciones, desde aquellas que presentan la mayor carga hasta aquellas que presentan la menor carga. Más adelante, se identifican los pedidos cuya(s) operación(es) inicial(es) (posiciones 1 a 2 en el orden de proceso), correspondan a cualquiera de los dos centros de trabajo más cargados. El propósito de tal identificación, consiste en seleccionar durante la fase relacionada con la generación de individuos, aquellos individuos cuyo ordenamiento de los pedidos, implique desde el inicio, la programación prioritaria de los centros de trabajo más cargados.

Paso 3. Obtención de la población de inicio.

Con base en el individuo descrito en la tabla 2, se obtienen de manera aleatoria 0,2l individuos, cuyo(s) pedido(s) inicial(es) (posiciones 1 a 2 en el orden del individuo), correspondan ya sea a uno o a dos, de aquellos pedidos identificados en el paso anterior. De este modo, la cantidad adicional de 0,8l individuos requeridos, se establece aleatoriamente, al evaluar el universo de soluciones. 


\section{Paso 4. Obtención y análisis de subpoblaciones.}

Se divide la población en seis subpoblaciones, una para cada objetivo. Cada subpoblación estará conformada por 1/6 individuos. Los individuos de cada subpoblación son evaluados y ordenados de menor valor a mayor valor. Posteriormente, se eliminan de cada subpoblación el $20 \%$ de aquellos individuos que presenten mayor valor. Para las seis subpoblaciones se requiere determinar en primer término el tiempo de proceso. La función de cálculo respectiva (makespan) se muestra en la ecuación 1:

FitnesS $_{M A K E S P A N}=\min \left\{\sum_{i=1}^{N} \sum_{j=1}^{M} P_{i j}\right\}$

En la ecuación 1, "N" representa el número de pedidos, "M" representa el número de máquinas y " $\mathrm{P}_{\mathrm{ij}}$ " hace referencia al tiempo del pedido i en la máquina j. Las subpoblaciones se evalúan utilizando métodos de penalización o de cálculo, así:

Método de penalización para el costo de mano de obra directa. En éste caso los puntos de penalización se asignan de acuerdo con las siguientes categorías: programa de trabajo diurno, programa de trabajo nocturno (10 pm a $6 \mathrm{am}$ ), programa de trabajo diurno en día dominical y programa de trabajo nocturno en día dominical (10 pm a 6 am). A cada uno de estos programas, le corresponde una asignación de puntos de penalización, la cual se encuentra fundamentada en los valores de recargo porcentual sobre el valor del salario en tiempo normal (ver tabla 4). Con el objetivo de establecer el valor de penalización por costo de mano de obra directa, se requiere determinar en primer término el comportamiento de la variable tiempo de proceso (horas).

Posteriormente, se identifica el esquema de organización del sistema de producción (jornadas laborales de 8 horas, de 16 horas o de 24 horas por día); después para cada intervalo de 8 horas dentro del intervalo de tiempo de procesamiento, se racionaliza la cantidad de personas requeridas. Una vez definido el número de personas requeridas por cada intervalo de 8 horas, se multiplica éste valor por el valor de la penalización correspondiente al programa de trabajo (diurno o nocturno en día hábil o en dominical) en el cual clasifique dicho intervalo; finalmente, se obtiene la penalización total por costo mano de obra directa, al sumar la penalización asignada a los diferentes intervalos de 8 horas que integran el horizonte de planeación en su integralidad.

Método de cuantificación de las emisiones equivalentes de dióxido de carbono. Para el caso, se evalúan tres tipos de fuentes: eléctrica (máquinas identificadas con las letras: A, B, C, D y E -ver tabla 5-; sistema de iluminación), gas (máquina identificada con la letra: $F$-ver tabla 5-) y diésel (montacargas). De manera consecuente, se obtiene el plan detallado de producción por hora del sistema de manufactura evaluado. En este plan, se especifica para cada una de las máquinas, su tiempo de procesamiento y su tiempo de espera. Posteriormente, se determina el total de energía consumida por máquina, al multiplicar los tiempos en mención por la potencia del equipo, ya sea en estado de procesamiento o en estado de espera. A su vez, en cuanto al sistema de iluminación, se procede a multiplicar su potencia (en horas diurnas o nocturnas), por el tiempo de trabajo diurno o nocturno, según el caso. Luego, se suma la energía eléctrica consumida, en sus diversos puntos de origen: máquinas identificas por las letras "A" a la "E" y sistema de iluminación.

Más adelante, la cantidad emitida de dióxido de carbono, se establece, multiplicando el consumo de energía eléctrica, por su respectivo factor equivalente de emisión de dióxido de carbono (ver tabla 6). Asimismo, para la máquina identificada con la letra " $F$ ", se procede de forma similar a la situación descrita, en cuanto a la definición correspondiente, a la emisión de dióxido de carbono, en aquellas máquinas que funcionan, por medio del consumo de energía eléctrica; salvo que en este caso, el consumo de energía, se afecta por el factor equivalente, aplicable al consumo de gas (ver tabla 6). Por último, para el montacargas que utiliza diésel, en su sistema de aprovisionamiento energético, se calcula su demanda de energía, al multiplicar el tiempo de recorrido de materiales por la potencia del equipo (montacargas); con posterioridad, a la cantidad de energía consumida, se le aplica aquel factor de emisión equivalente, definido para las fuentes a base de tal tipo de combustible (Ver tabla 6).

Método de penalización para la accidentalidad laboral. El método se encuentra basado en la información histórica de la Compañía, relacionada con la probabilidad de accidentalidad laboral por hora (ver tabla 7). Con el objetivo de establecer el valor de penalización por accidentalidad, se requiere determinar en primer término el comportamiento de la variable tiempo de proceso (en horas); enseguida, se identifica el esquema de organización del sistema de producción (jornadas de: 8 horas, 16 horas o 24 horas); después, a cada hora de la jornada laboral dentro del intervalo de tiempo de proceso, se le asigna la respectiva probabilidad de accidentalidad laboral (ver tabla 7); más adelante, este valor se multiplica por 100, generándose el 
número de puntos que serán aplicados como valor de penalización a cada hora; finalmente, los valores de penalización obtenidos por hora, pueden sumarse uno a uno hasta obtener el valor de la penalización total.

Método de penalización para la intensidad del sonido. En este caso, se determina en primera instancia, el plan detallado de producción, bajo el cual puede observarse el comportamiento horario del horizonte de planeación. Después, para cada hora de labor se establece el nivel de intensidad sonora percibido en un punto particular de la planta de producción (ver tabla 8). Una vez establecido el valor anterior, se obtiene su equivalencia matemática como proporción de 100 puntos para 85 decibeles. Es así como, esta equivalencia constituye la penalización asignable a cada hora de labor. Con posterioridad, se define la penalización total, procediendo a sumar las penalizaciones horarias a lo largo del horizonte de planeación. Por último, se identifica la respectiva penalización promedio turno (un turno equivale a 8 horas de labor), al dividir el nivel de penalización total, entre el número de turnos que integran tal horizonte de planeación. La expresión utilizada para el cálculo de la intensidad sonora, se aprecia enseguida:

$$
\left.P S P=10 \log \left(\sum_{I=1}^{N} 10^{\frac{L p i}{10}}\right)\right)
$$

Donde: $\mathrm{PSP}=$ Promedio sonoro en el punto $\mathrm{P} ; \mathrm{N}=$ Número de fuentes de las cuales proveniene cada nivel de intensidad sonora; $\mathrm{Lpi}=$ Nivel sonoro medido para cada fuente de manera individual, en el punto P. Al aplicar la expresión para el cálculo de PSP, se observaría que a mayor número de fuentes operando de manera simultánea, el promedio sonoro (PSP) en el punto $\mathrm{P}$, sería mayor a la intensidad sonora medida para cada una de las fuentes de manera individual, en el punto $P$.

Método de cuantificación para el peso manipulado en promedio por hora. Para el caso, se determina con antelación, el plan horario de producción. De esta forma, se procede a sumar los pesos correspondientes a aquellas piezas manipuladas cada hora, en los diferentes puestos de trabajo. Posteriormente, se suman los pesos detectados por hora, durante la totalidad de horas que integran el horizonte de planeación. Finalmente, el peso total establecido, se divide entre el número de horas que integran el horizonte de planeación evaluado.

\section{Paso 5. Definir la población número uno.}

La población número uno, se conforma así: cada subpoblación de 0,81/6 individuos, se segmenta en seis intervalos. De cada intervalo se extraen aleatoriamente 0,0139l individuos. En total, las subpoblaciones aportan $0,5 \mathrm{I}$ individuos a la nueva población. A los anteriores $0,5 \mathrm{l}$ individuos se les aplica con una probabilidad de $96 \%$, la operación de cruzamiento. Esta operación se realiza, hasta estimar otro $30 \%$ del tamaño de la población (I). El cruzamiento se ejecuta intercambiando posiciones entre pedidos. El siguiente $5 \%$ de los individuos, se origina en la mutación aleatoria de los anteriores 0,8 I individuos. Otro $5 \%$ de los individuos, se selecciona de aquellos individuos, cuyos pedidos iniciales (posiciones 1 a 2 en el orden del individuo), corresponden, ya sea a uno a dos de aquellos pedidos identificados en el paso 2. Mientras que el último $10 \%$ de individuos, se obtiene de la exploración aleatoria del total de posibles soluciones.

\section{Paso 6. Estructurar por frentes de Pareto.}

La población se evalúa en las seis funciones objetivo (ver actividad № 1 en la figura 3); más adelante, se toma cada individuo, procediendo a comparar los valores establecidos en cada una de sus funciones, respecto a los valores estimados para los otros individuos en las mismas funciones (ver actividad № 2 en la figura 3). De esta manera, se declara a un individuo como "no dominado", cuando el individuo en cuestión presenta menores valores en todas sus funciones, con relación a los valores tomados por ciertos individuos en estas mismas funciones (ver actividad № 3 en la figura 3 ).

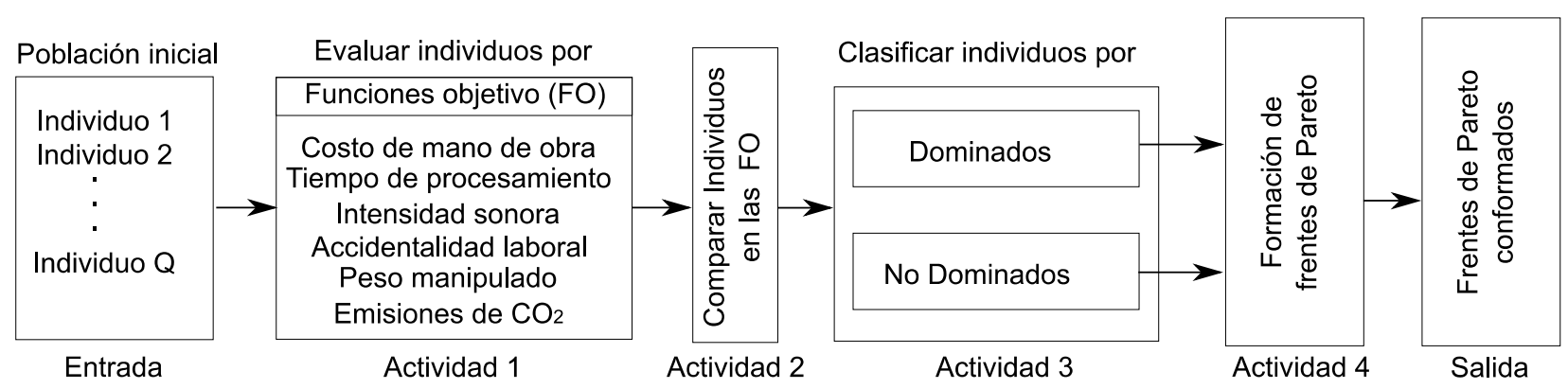

Fig. 3: Conformación de frentes de Pareto. 
De acuerdo con lo descrito, el proceso de comparación correspondiente a los demás individuos de la población, continúa ejecutándose hasta definir el primer grupo de "no dominados" (primer frente de Pareto) (ver actividad № 4 en la figura 3). Enseguida, se repiten las actividades anteriores para aquellos individuos no categorizados en el Primer frente de Pareto; es así como, se logra identificar el segundo frente de Pareto (ver actividad № 4 en la figura 3). Consecuentemente, el método de clasificación expuesto, se aplica de nuevo sobre los individuos dominados, hasta caracterizar la totalidad de frentes de Pareto asignables a la población analizada.

\section{Paso 7. Obtener la población número dos.}

A esta población ingresan los individuos agrupados por frentes de la población uno, hasta completar $0,5 \mathrm{I}$ individuos. Asimismo, los otros $0,5 \mathrm{I}$ individuos se obtienen de la siguiente forma: se define el porcentaje de individuos por frente. Cada valor porcentual identificado, se aplica a 0,31 individuos. La cantidad de individuos obtenidos constituye la cantidad de nuevos individuos que se originan a partir del cruzamiento de los individuos que constituyen cada frente. Posteriormente, para los 0,8I individuos obtenidos, se aplica la operación de mutación. De ésta manera, el siguiente $5 \%$ de los individuos, se origina en la mutación aleatoria de los anteriores $0,8 \mathrm{l}$ individuos. Otro $5 \%$ de los individuos, se selecciona de aquellos individuos, cuyos pedidos iniciales (posiciones 1 a 2 en el individuo), corresponden ya sea a uno o a dos de aquellos pedidos identificados en el paso 2. Mientras que el último $10 \%$ de individuos, se obtiene de la exploración aleatoria del universo de soluciones. Una vez obtenida la población número dos, se repite desde el paso cuatro hasta completar 100 iteraciones. Es así como, al terminar las 100 iteraciones, se procede a ordenar la población final por frentes, de acuerdo con lo establecido en el paso seis de la metodología.

\section{Paso 8. Realizar búsqueda aleatoria en el primer frente de Pareto.}

En este paso, se pretende diversificar el primer frente de Pareto, por medio de la búsqueda de nuevos individuos no dominados. Para este efecto, se seleccionan de manera aleatoria, una cantidad equivalente al $20 \%$ de los individuos que integran el frente bajo estudio. Es así como, se procede a mutar cualquiera de los campos de cada individuo elegido, durante un máximo de 11 oportunidades. Sí al ejecutarse las 11 iteraciones, no se logran identificar nuevos individuos de interés, el procedimiento en mención finaliza para aquel individuo analizado. De este modo, continúa la evaluación del siguiente individuo, hasta completar la revisión de aquellos individuos que fueron seleccionados.

\section{Paso 9. Aplicar la función lineal de sumas ponderadas.}

En algunos casos particulares, durante la presentación de resultados se requiere establecer la mayor importancia de cierto individuo, respecto a otro u otros individuos. Con este propósito, se utilizará la siguiente función lineal para sumas ponderadas:

$P\left(X_{j}\right)_{i}=\left[\frac{F P\left(f_{i}\right)}{\sum_{k=1}^{M} F P\left(f_{k}\right)}\right]\left[\frac{f_{i}^{\text {max }}-f_{i}\left(x_{j}\right)}{f_{i}^{\text {max }}-f_{i}^{\text {min }}}\right]$

Donde: $f_{i}{ }^{\max }=$ Valor máximo encontrado para la función en una población; $\mathrm{f}_{\mathrm{i}}$ min $=$ Valor mínimo encontrado para la función en una población; $f_{i}\left(x_{j}\right)=$ Valor de la función $f_{i}$ para el individuo $X_{j}$; $F P=F a c t o r ~ d e$ Ponderación; FP( $\left.f_{i}\right)$ = Factor de Ponderación de fi (este valor es definido por el agente decisor de acuerdo con el tipo de contexto analizado y varía de 0 a 100); $\operatorname{FP}\left(f_{k}\right)=$ Factor de Ponderación total (la sumatoria de éste factor es 1); $\mathrm{M}=$ Número de Funciones Objetivo; $\mathrm{P}\left(\mathrm{X}_{\mathrm{j}}\right)_{\mathrm{i}}=$ Ponderación del Individuo en la Función Objetivo; $X_{j}=$ Individuo de la población. Una vez calculado $P\left(X_{j}\right)$ i, se obtiene:

$P\left(X_{j}\right)=\sum_{i=1}^{M} P\left(X_{j}\right)_{i}$

Donde: $M=$ Número de Funciones Objetivo; $P(X j)=$ Factor de ponderación lineal del individuo “j”; $P(X j) i=$ Ponderación del Individuo en la Función Objetivo; Xj = Individuo de la población.

\section{Paso 10. Presentar la búsqueda de literatura en programación sostenible}

En este paso, se presenta la síntesis de la revisión sistemática de literatura, en programación de sistemas Job Shop, bajo un enfoque de sostenibilidad. Con relación a lo anterior, se procede a definir las ecuaciones de búsqueda necesarias y a ejecutar en consecuencia, las respectivas consultas en fuentes pertinentes. Más adelante, se estructura una matriz para análisis de información, la cual permite clasificar las variables tratadas en cada artículo revisado, de acuerdo con su naturaleza económica, ambiental o social. Finalmente, se comparan los desarrollos académicos evaluados con aquellas orientaciones específicas del presente artículo. 
Paso 11. Comparación del método diseñado con el método de operación actual.

Con este propósito, se determinan las características actuales de operación, en lo referente a la definición del programa de producción por parte de la empresa analizada. Asimismo, se identifica por medio de la evaluación del conjunto de respuestas obtenidas después de aplicar el método propuesto, aquel individuo que presente el menor tiempo de procesamiento (expresado en horas). De este modo, se establece en consecuencia, cuál sería el impacto de implementar, el mencionado individuo de menor tiempo de proceso, sobre el desempeño de las otras variables de interés.

\section{Paso 12. Comparación del método diseñado con la programación por reglas de prioridad.}

Para la situación específica, se realiza en primer término la secuenciación de producción, utilizando con este propósito, las reglas de programación relacionadas enseguida: LPT, SOT, FCFS y LCFS. Una vez, diseñados los programas de producción, se procede a estimar para cada regla, el comportamiento de la variable: "factor ponderado". Por último, se efectúan las comparaciones de los valores tomados por la variable en cuestión, respecto al valor definido para la misma variable, después de implementar el método propuesto.

\section{Paso 13. Comparación del método diseñado con el método de "cuello de botella móvil".}

Con este objetivo, se procede a identificar en primera instancia el conjunto de operaciones que integran los cuellos de botella móviles (Goldratt y Cox, 2005). Posteriormente, se determina por cada pedido el comportamiento de la variable "factor restricción". Más adelante, se ordenan los pedidos a nivel descendente, tomando para el caso como criterio de clasificación, el valor calculado de la variable "factor restricción". Luego, se obtiene el respectivo programa de producción, siguiendo para este efecto los lineamientos de la secuencia ya establecida. Después, se define el valor correspondiente a la variable "factor ponderado". Finalmente, se compara el último valor con el valor detectado para la misma variable, tras aplicar el método propuesto.

Paso 14. Confiabilidad del algoritmo genético a nivel de experimentación.

El programa se ejecuta durante 30 oportunidades, estableciendo cada vez el comportamiento promedio de los seis parámetros evaluados. A partir de lo anterior, se realiza análisis de varianza (dos tratamientos y 15 repeticiones por tratamiento), al valor promedio de cada variable. De acuerdo con lo expuesto, se procede a definir bajo un nivel de confianza de $95 \%$, la estabilidad estadística de los datos. De este modo, se pretende determinar la confiabilidad de aquellos resultados obtenidos, al implementar el algoritmo propuesto.

\section{EXPERIMENTACIÓN}

Para éste efecto, se procedió a evaluar el área de trabajo, en la cual se manufacturan partes a medida (base torres, parales), a ser utilizadas en el proceso correspondiente, a la instalación de torres de transmisión de energía.

\section{Datos utilizados para la determinación del tiempo de procesamiento.}

En la tabla 3, se registran los datos correspondientes a la ruta de proceso por pedido y su tiempo de operación en cada centro de trabajo. Cada centro cuenta con una máquina y cada máquina requiere un operario para su manejo y control continuo. De acuerdo con el sistema de producción, se observa que cada pedido sigue una ruta de fabricación diferente. Los centros de trabajo son: $A, B, C, D, E$ y $F$.

Tabla 3: Rutas y tiempos de operación por pedido (en horas).

\begin{tabular}{|c|l|r|l|r|r|r|r|r|l|r|r|r|}
\hline Pedidos & \multicolumn{10}{|c|}{ Rutas tiempos de operación } \\
\hline 1 & $\mathrm{E}$ & 12 & $\mathrm{~B}$ & 9 & $\mathrm{~F}$ & 18 & $\mathrm{~A}$ & 14 & $\mathrm{C}$ & 15 & $\mathrm{D}$ & 10 \\
\hline 2 & $\mathrm{~B}$ & 14 & $\mathrm{E}$ & 12 & $\mathrm{~A}$ & 15 & $\mathrm{D}$ & 8 & $\mathrm{C}$ & 12 & $\mathrm{~F}$ & 11 \\
\hline 3 & $\mathrm{E}$ & 16 & $\mathrm{~A}$ & 12 & $\mathrm{C}$ & 11 & $\mathrm{~F}$ & 10 & $\mathrm{D}$ & 12 & $\mathrm{~B}$ & 13 \\
\hline 4 & $\mathrm{C}$ & 14 & $\mathrm{D}$ & 12 & $\mathrm{E}$ & 17 & $\mathrm{~A}$ & 10 & $\mathrm{~F}$ & 11 & $\mathrm{~B}$ & 15 \\
\hline 5 & $\mathrm{C}$ & 12 & $\mathrm{~F}$ & 11 & $\mathrm{E}$ & 13 & $\mathrm{D}$ & 15 & $\mathrm{~B}$ & 10 & $\mathrm{~A}$ & 7 \\
\hline 6 & $\mathrm{~F}$ & 12 & $\mathrm{~B}$ & 14 & $\mathrm{E}$ & 11 & $\mathrm{~A}$ & 17 & $\mathrm{C}$ & 10 & $\mathrm{D}$ & 9 \\
\hline 7 & $\mathrm{C}$ & 14 & $\mathrm{~F}$ & 11 & $\mathrm{D}$ & 17 & $\mathrm{~A}$ & 14 & $\mathrm{~B}$ & 17 & $\mathrm{E}$ & 16 \\
\hline 8 & $\mathrm{~F}$ & 14 & $\mathrm{~B}$ & 15 & $\mathrm{C}$ & 17 & $\mathrm{E}$ & 15 & $\mathrm{D}$ & 16 & $\mathrm{~A}$ & 14 \\
\hline 9 & $\mathrm{E}$ & 11 & $\mathrm{D}$ & 14 & $\mathrm{~A}$ & 18 & $\mathrm{~F}$ & 17 & $\mathrm{~B}$ & 10 & $\mathrm{C}$ & 14 \\
\hline
\end{tabular}


Datos utilizados en la determinación de la penalización por costos de mano de obra directa.

La información utilizada para obtener la penalización por costos mano de obra se relaciona en la tabla 4. Los datos correspondientes a aquellas ponderaciones que permiten determinar el nivel salarial como valor adicional al costo del salario en tiempo normal, se tomaron del respectivo código laboral.

Tabla 4: Ponderaciones sobre el tiempo normal y penalización.

\begin{tabular}{|l|c|c|c|c|c|}
\hline $\begin{array}{c}\text { Programación } \\
\text { trabajo }\end{array}$ & $\begin{array}{c}\text { Ponderación } \\
\text { día hábil }\end{array}$ & $\begin{array}{c}\text { Penalización } \\
\text { (Puntos) }\end{array}$ & $\begin{array}{c}\text { Ponderación } \\
\text { día festivo }\end{array}$ & $\begin{array}{c}\text { Penalización } \\
\text { (puntos) }\end{array}$ & Horario \\
\hline Diurno. & 0 & 0 & 0,75 & 75 & 6 am a 10 pm \\
\hline Nocturno. & 0,35 & 35 & 1,10 & 100 & 10 pm a 6 am \\
\hline
\end{tabular}

Datos utilizados en la determinación de las emisiones equivalentes de dióxido de carbono.

En la tabla 5, se aprecia la información correspondiente a cada una de las máquinas o sistemas de apoyo a la producción que fueron analizados.

Tabla 5: Potencia de las máquinas de procesamiento o de los sistemas de apoyo a producción.

\begin{tabular}{|c|c|c|c|c|c|c|c|c|c|}
\hline \multirow[t]{2}{*}{ Máquina } & \multirow{2}{*}{$\begin{array}{l}\text { Fuente } \\
\text { energía }\end{array}$} & \multirow{2}{*}{$\begin{array}{c}\text { Potencia } \\
\text { proceso } \\
(\mathrm{Kw})\end{array}$} & \multirow{2}{*}{$\begin{array}{c}\text { Potencia } \\
\text { espera } \\
(\mathrm{Kw})\end{array}$} & \multirow{2}{*}{$\begin{array}{l}\text { Máquina o } \\
\text { sistema } \\
\text { apoyo }\end{array}$} & \multirow[t]{2}{*}{ Fuente } & \multicolumn{2}{|c|}{ Potencia (Kw) } & \multicolumn{2}{|c|}{ Potencia (Kw) } \\
\hline & & & & & & Proceso & Espera & $\begin{array}{l}6 \mathrm{am} \\
6 \mathrm{pm}\end{array}$ & $\begin{array}{l}6 \mathrm{pm} \\
6 \mathrm{am}\end{array}$ \\
\hline A & Eléctrica & 9.2 & 0.368 & $E$ & Eléctrica & 11.24 & 0.45 & & \\
\hline$B$ & Eléctrica & 9.2 & 0.368 & $F$ & Gas & 93.04 & 3.72 & & \\
\hline $\mathrm{C}$ & Eléctrica & 5.1 & 0.204 & Montacargas & Diésel & 34 & $x$ & & \\
\hline$D$ & Eléctrica & 2.5 & 0.1 & Iluminación & Eléctrica & & & 2.4 & 4.8 \\
\hline
\end{tabular}

Respecto a la información anterior, se aclara que en la situación bajo análisis, el montacargas se utiliza solamente para realizar 9 viajes, los cuales cubren aquellos trayectos existentes, desde los sitios correspondientes a la finalización de cada pedido (localización de cada tipo de máquina, según el número asignado al pedido), hasta aquel sitio de ubicación del almacén de producto terminado. A su vez, se señala que cada viaje tiene en promedio una duración de 2 minutos. Finalmente, se anota que tal sistema de manejo de materiales, presenta otro tipo de usos (manejo de materiales en: las diferentes secciones de producción, entre secciones de producción, bodega de materia prima, almacén de producto terminado y patios de maniobras), cuya cuantificación se encuentra por fuera del contexto aquí evaluado.

De manera complementaria, se exponen en la tabla 6, los factores establecidos para la determinación de los niveles de emisión equivalentes de dióxido de carbono, de acuerdo con el tipo de fuente (Ministerio de Minas y Energía, 2015):

Tabla 6: Factores de emisión equivalentes para dióxido de carbono.

\begin{tabular}{|l|c|}
\hline Fuente & Factor $\left(\mathrm{Kg} \mathrm{CO}_{2} / \mathrm{Kwh}\right)$ \\
\hline Eléctrica & 0.3740 \\
\hline Gas & 0.2018 \\
\hline Diésel & 0.2495 \\
\hline
\end{tabular}

Datos utilizados en la determinación de la accidentalidad laboral.

Los datos relacionados con la probabilidad de ocurrencia de accidentes de trabajo, a lo largo de la jornada laboral y sus puntos de penalización, se presentan en la tabla 7. La información suministrada, se recolectó y se sistematizó, en el área de gestión humana de la Compañía objeto de estudio.

Datos utilizados en la determinación del nivel de intensidad sonora.

Al respecto, se midió la intensidad sonora percibida en determinado punto de la planta de producción para cada equipo operando de forma individual, es decir, sólo se permitía que un equipo estuviera en funcionamiento, mientras los demás equipos debían permanecer apagados. El punto de medición elegido es equidistante a los diversos centros de trabajo y se encuentra localizado al interior de la sección analizada. Las mediciones establecidas, se ilustran en la tabla 8. Estas mediciones constituyen los valores correspondientes a la variable Lpi en la ecuación $N^{\circ} 2$. 
Tabla 7: Probabilidad de accidentalidad laboral y penalización.

\begin{tabular}{|c|c|c|c|c|c|c|c|c|c|}
\hline \multirow[t]{2}{*}{ Horario } & \multicolumn{2}{|c|}{$\begin{array}{c}\text { Probabilidad } \\
\text { accidentalidad } \\
\text { Laboral }\end{array}$} & \multicolumn{2}{|c|}{$\begin{array}{l}\text { Penalidad } \\
\text { (puntos) }\end{array}$} & \multirow[t]{2}{*}{ Horario } & \multicolumn{2}{|c|}{$\begin{array}{l}\text { Probabilidad } \\
\text { accidentalidad } \\
\text { laboral }\end{array}$} & \multicolumn{2}{|c|}{$\begin{array}{l}\text { Penalidad } \\
\text { (puntos) }\end{array}$} \\
\hline & am & $\mathrm{Pm}$ & am & $\mathrm{Pm}$ & & $\mathrm{Pm}$ & am & $\mathrm{pm}$ & am \\
\hline 06:00 a 08:00 & 0.01 & 0.03 & 1 & 3 & $12: 00$ a $02: 00$ & 0.02 & 0.03 & 2 & 3 \\
\hline 08:00 a 10:00 & 0.01 & 0.04 & 1 & 4 & $02: 00$ a $04: 00$ & 0.01 & 0.04 & 1 & 4 \\
\hline $10: 00$ a $12: 00$ & 0.02 & 0.02 & 2 & 2 & 04:00 a 06:00 & 0.02 & 0.04 & 2 & 4 \\
\hline
\end{tabular}

Tabla 8: Intensidad sonora percibida en un punto "P" (db).

\begin{tabular}{|l|c|c|c|c|c|c|}
\hline Máquina & A & B & C & D & E & F \\
\hline Intensidad sonora $(\mathrm{db})$ & 95 & 85 & 86 & 95 & 90 & 100 \\
\hline
\end{tabular}

Datos utilizados en la determinación del peso promedio manipulado por hora en los centros de trabajo.

Con este propósito, la empresa suministró la información relacionada con el peso promedio del tipo de producto manipulado en cada pedido, la cual se obtuvo estableciendo el peso de cada artículo, al ingresar a cada uno de los centros de trabajo. Esta información se presenta en la tabla 9:

Tabla 9: Peso promedio de cada trabajo por pedido $(\mathrm{Kg})$.

\begin{tabular}{|l|c|c|c|c|c|c|c|c|c|}
\hline Pedido & 1 & 2 & 3 & 4 & 5 & 6 & 7 & 8 & 9 \\
\hline Peso promedio pieza en proceso $(\mathrm{Kg})$ & 47.5 & 51.3 & 42.7 & 62.1 & 56.8 & 60.7 & 58.3 & 43.4 & 59.5 \\
\hline
\end{tabular}

\section{RESULTADOS Y DISCUSION}

\section{Población final obtenida por medio de la aplicación del método propuesto.}

De acuerdo con los pasos 1 a 9 de la metodología, se presenta en la tabla 10 el resumen de resultados para 3 individuos seleccionados de una población total de 100 individuos. También se muestra para la totalidad de individuos, el comportamiento de las variables analizadas en cuanto a los parámetros: promedio, valor mínimo y valor máximo. Los valores máximos y mínimos, registrados en la mencionada tabla, permiten concluir que existe una amplia variedad de soluciones. De manera específica, al comparar la solución número 13 con la solución número 32, en lo que respecta a las diversas variables, se establece que: la primera supera el desempeño de la segunda, a nivel de tiempo de proceso (en horas y en semanas), al igual que en emisiones equivalentes de dióxido de carbono; obtienen el mismo valor, en cuanto al comportamiento del parámetro costos de mano de obra directa y finalmente, la segunda supera el desempeño de la primera, al observar los valores tomados por las otras variables de interés (accidentalidad laboral, nivel de intensidad sonora y peso manipulado en promedio, durante cada turno).

Tabla 10: Resumen de resultados.

\begin{tabular}{|c|c|c|c|c|c|c|c|c|c|}
\hline $\begin{array}{c}\text { Posición } \\
\text { individuo } \\
\text { en la } \\
\text { población }\end{array}$ & $\begin{array}{c}\text { Tiempo } \\
\text { proceso } \\
\text { (horas) }\end{array}$ & $\begin{array}{c}\text { Horas } \\
\text { en el } \\
\text { día }\end{array}$ & $\begin{array}{c}\text { Días en } \\
\text { la } \\
\text { Semana }\end{array}$ & $\begin{array}{c}\text { Tiempo } \\
\text { proceso } \\
\text { (semanas) }\end{array}$ & $\begin{array}{c}\text { Penalidad } \\
\text { costos } \\
\text { mano } \\
\text { obra } \\
\text { (puntos) }\end{array}$ & $\begin{array}{c}\text { Emisiones } \\
\text { equivalentes } \\
\text { dióxido de } \\
\text { carbono } \\
\text { (Kg) }\end{array}$ & $\begin{array}{c}\text { Penalidad } \\
\text { accidentes } \\
\text { laborales } \\
\text { (puntos) }\end{array}$ & $\begin{array}{c}\text { Penalidad } \\
\text { nivel de } \\
\text { intensidad } \\
\text { sonora } \\
\text { (puntos) }\end{array}$ & $\begin{array}{c}\text { Peso } \\
\text { promedio } \\
\text { turno } \\
\text { (Kg) }\end{array}$ \\
\hline 2 & 185 & 24 & 7 & 1.1 & 3055 & 4134.02 & 1681 & 934.34 & 1659.65 \\
\hline 13 & 166 & 16 & 6 & 1.73 & 0 & 4019.46 & 1362 & 947.61 & 1849.61 \\
\hline 32 & 239 & 8 & 6 & 4.98 & 0 & 4129.05 & 1053 & 891.14 & 1284.67 \\
\hline Promedio & 208.27 & & & 1.91 & 1333.92 & 4135.67 & 1500 & 918.08 & 1486.67 \\
\hline Mínimo & 160 & & & 0.95 & 0 & 4007.38 & 1024 & 870.34 & 1171.90 \\
\hline Máximo & 262 & & & 4.98 & 3600 & 4243.23 & 1727 & 951.41 & 1918.97 \\
\hline
\end{tabular}


A partir de la revisión de la tabla 10, se obtiene la clasificación general de individuos por frentes de Pareto. Esta clasificación, se señala enseguida: 32 individuos en el frente 1 (individuos no dominados), 15 individuos en el frente 2, 11 individuos en el frente 3,10 individuos en el frente 4, 8 individuos en el frente 5, 7 individuos en el frente 6, 5 individuos en el frente 7, 5 individuos en el frente 8,4 individuos en el frente 9 y 3 individuos en el frente 10.

Con el objetivo de sistematizar la información registrada en el primer frente de Pareto, el comité de dirección de la compañía, procedió a efectuar la clasificación por intervalos de los datos correspondientes, a la variable tiempo de procesamiento. Para éste fin, el mencionado comité, ejecutó las actividades descritas enseguida: en una primera sesión, se analizó la información en cuestión y se establecieron en términos generales, aquellas circunstancias organizacionales, que implicarían la necesidad de utilizar cierta clase de datos; con posterioridad, cada uno de los integrantes del equipo de dirección, categorizó de manera individual, los diversos datos de interés y por último, durante una segunda sesión, se presentaron las clasificaciones obtenidas por persona, lo cual permitió generar intercambio de opiniones y, asimismo el respectivo consenso, en referencia a la agrupación por intervalos de la información suministrada (datos de la variable tiempo de procesamiento). En la tabla 11, se aprecia la clasificación por intervalos de la variable tiempo de procesamiento, definida por el equipo directivo de la empresa, objeto de estudio.

Tabla 11: Intervalos para el tiempo de procesamiento (en semanas).

\begin{tabular}{|c|c|c|l|c|}
\hline $\begin{array}{c}\text { Número } \\
\text { Intervalo }\end{array}$ & $\begin{array}{c}\text { Límite } \\
\text { Inferior } \\
\text { (semanas) }\end{array}$ & $\begin{array}{c}\text { Límite } \\
\text { Superior } \\
\text { (semanas) }\end{array}$ & $\begin{array}{c}\text { Números de } \\
\text { identificación de } \\
\text { los individuos del } \\
\text { primer frente. }\end{array}$ \\
\hline 1 & 0,0 & 1.5 & $\begin{array}{l}\text { En este caso, se busca realizar entregas rápidas, debido } \\
\text { a que los clientes de acuerdo con sus necesidades } \\
\text { particulares, pueden requerir atención inmediata. Además, } \\
\text { existen periodos económicos caracterizados por el } \\
\text { crecimiento permanente de la demanda. En la situación } \\
\text { anterior, coexisten una gran variedad de compromisos, a } \\
\text { pesar de lo cual, el plan de entregas debe ejecutarse, } \\
\text { cumpliendo las fechas previamente establecidas. }\end{array}$ & 1 a 9 \\
\hline 2 & 1,5 & 3.0 & $\begin{array}{l}\text { En éste caso, la proporción de pedidos urgentes que } \\
\text { requieren los clientes, se reduce de manera significativa. }\end{array}$ & $\begin{array}{l}\text { Por tanto, se debe mantener un excelente nivel de } \\
\text { servicio para los diversos pedidos programados. } \\
\text { Igualmente, los periodos económicos presentan } \\
\text { estabilidad estructural, lo cual conduce a que se efectúen } \\
\text { pocos cambios en las asignaciones de manufactura. }\end{array}$ \\
\hline 3 & 3,0 & 5.0 & $\begin{array}{l}\text { En éste caso, los periodos económicos presentan } \\
\text { características de recesión; por tanto, la asignación de los } \\
\text { recursos utilizados en la ejecución del programa de } \\
\text { producción, conduce a que se obtengan tiempos de } \\
\text { entrega significativamente altos. Respecto a lo anterior, } \\
\text { cabe aclarar que los mencionados tiempos de entrega, } \\
\text { también corresponden a las necesidades de entrega de } \\
\text { las compañías cliente. }\end{array}$ & 23 a 32 \\
\hline
\end{tabular}

Para cada intervalo definido, debe establecerse cuál individuo en particular, presenta la potencialidad de convertirse en la mejor solución. A partir de lo expuesto, se argumenta que se seleccionará, en cada uno de los intervalos nombrados, aquel individuo cuyo valor de la función lineal obtenida, por medio de la aplicación de la ecuación № $4\left(\mathrm{P}\left(\mathrm{X}_{\mathrm{j}}\right)\right)$, corresponda al mayor valor de tal función. Con este objetivo, se asignará un factor de ponderación de 33,33\% ( $\mathrm{FP}\left(\mathrm{f}_{\mathrm{i}}\right)$ en la ecuación $\left.\mathrm{N}^{\circ} 3\right)$, a cada grupo de variables evaluadas (económicas, sociales, ambientales). En este sentido, para cada variable de interés, el comité de dirección de la compañía, definió las siguientes participaciones porcentuales: 16,66 \% (tiempo de proceso en semanas), 16,66 \% (costos de mano de obra directa), 33,33\% (emisiones equivalentes de dióxido de carbono), $10 \%$ (nivel de intensidad sonora), 18,33 \% (accidentalidad laboral) y $5 \%$ (peso manipulado en promedio). Al evaluar en la ecuación № 3, los anteriores valores ponderados; se determinaron los siguientes individuos, como aquellos individuos de mejor comportamiento, por intervalo.

Complementariamente, se presenta en la tabla 13 la información relacionada con los puntos mínimos de cada variable, los cuales podrían implementarse, en algunos contextos de operación de la compañía analizada. Al respecto, se aclara que en caso de encontrar dos o más individuos con igual valor mínimo, se seleccionará aquel individuo con mayor valor de factor ponderado $\left(\left(P\left(X_{j}\right)\right)\right.$, en el conjunto correspondiente a la población de respuesta (100 individuos). 
Tabla 12: Individuos de mejor comportamiento por intervalo.

\begin{tabular}{|c|c|c|c|c|c|c|c|c|}
\hline $\begin{array}{c}\mathrm{N}^{\circ} \\
\text { Intervalo }\end{array}$ & $\begin{array}{c}\mathrm{N}^{\circ} \\
\text { Individuo } \\
\text { elegido }\end{array}$ & $\begin{array}{c}\text { Tiempo } \\
\text { proceso } \\
\text { (semanas) }\end{array}$ & $\begin{array}{c}\text { Penalidad } \\
\text { costos mano } \\
\text { obra (puntos) }\end{array}$ & $\begin{array}{c}\text { Emisiones } \\
\text { equivalentes } \\
\mathrm{CO}_{2}(\mathrm{Kg})\end{array}$ & $\begin{array}{c}\text { Penalidad } \\
\text { accidentes } \\
\text { laborales } \\
\text { (puntos) }\end{array}$ & $\begin{array}{c}\text { Penalidad } \\
\text { nivel de } \\
\text { intensidad } \\
\text { sonora } \\
\text { (puntos) }\end{array}$ & $\begin{array}{c}\text { Peso } \\
\text { promedio } \\
\text { turno } \\
(\mathrm{Kg})\end{array}$ & $\begin{array}{c}\text { Factor } \\
\text { ponderado } \\
\text { individuo } \\
\text { elegido } \\
\left(\mathrm{P}\left(\mathrm{X}_{\mathrm{j}}\right)\right)\end{array}$ \\
\hline 1 & 9 & 1.42 & 675 & 4011.41 & 1365 & 951.41 & 1894.83 & $68.54 \%$ \\
\hline 2 & 12 & 1.66 & 675 & 4057.12 & 1359 & 933.66 & 1650.34 & $65.11 \%$ \\
\hline 3 & 28 & 4.40 & 0 & 4094 & 1024 & 916.47 & 1455.14 & $66.66 \%$ \\
\hline
\end{tabular}

Tabla 13: Puntos de mínimo valor.

\begin{tabular}{|l|c|c|c|c|c|c|c|}
\hline \multicolumn{1}{|c|}{$\begin{array}{l}\text { Variable de } \\
\text { mínimo valor }\end{array}$} & $\begin{array}{c}N^{\circ} \\
\text { Individuo }\end{array}$ & $\begin{array}{c}\text { Tiempo } \\
\text { proceso } \\
\text { (semanas) }\end{array}$ & $\begin{array}{c}\text { Penalidad } \\
\text { costos } \\
\text { mano obra } \\
\text { (puntos) }\end{array}$ & $\begin{array}{c}\text { Emisiones } \\
\text { equivalentes } \\
\text { dióxido de } \\
\text { carbono } \\
(\text { Kg) }\end{array}$ & $\begin{array}{c}\text { Penalidad } \\
\text { accidentes } \\
\text { laborales } \\
\text { (puntos) }\end{array}$ & $\begin{array}{c}\text { Penalidad } \\
\text { nivel de } \\
\text { intensidad } \\
\text { sonora } \\
\text { (puntos) }\end{array}$ & $\begin{array}{c}\text { Peso } \\
\text { promedio turno } \\
\text { (Kg) }\end{array}$ \\
\hline $\begin{array}{l}\text { Tiempo } \\
\text { proceso }\end{array}$ & 1 & 0.95 & 3020 & 4074.69 & 1685 & 950.81 & 1917.97 \\
\hline $\begin{array}{l}\text { Penalidad } \\
\text { Costos }\end{array}$ & $\begin{array}{l}\text { No }^{\circ} \text { 13 (y } \\
14 \text { más) }\end{array}$ & 1.73 & 0 & 4019.46 & 1362 & 947.61 & 1849.61 \\
\hline $\begin{array}{l}\text { Emisiones } \\
\text { dióxido } \\
\text { carbono }\end{array}$ & 11 & 1.66 & 0 & 4007.38 & 1362 & 950.81 & 1918.97 \\
\hline $\begin{array}{l}\text { Penalidad } \\
\text { accidentes } \\
\text { Laborales }\end{array}$ & 28 & 4.40 & 0 & 4094.22 & 1024 & 916.47 & 1455.14 \\
\hline $\begin{array}{l}\text { Penalidad } \\
\text { Intensidad } \\
\text { sonora }\end{array}$ & 22 & 2.73 & 0 & 4157.65 & 1365 & 870.34 & 1171.89 \\
\hline $\begin{array}{l}\text { Peso promedio } \\
\text { manipulado }\end{array}$ & 22 & 2.73 & 0 & 4157.65 & 1365 & 870.34 & 1171.89 \\
\hline
\end{tabular}

Análisis del estado de avance de la programación de los sistemas Job Shop, bajo enfoque sostenible.

Al revisar los lineamientos del paso 10 de la metodología, se estructuraron dos ecuaciones de búsqueda, las cuales se aprecian enseguida: "Job Shop" and sustainability y, "Job Shop" and sustainable. De esta forma, se ejecutaron las respectivas consultas, en las bases de datos presentadas a continuación: scopus, web of science, scielo, academic search complete, business source complete y science direct.

De acuerdo con lo planteado, la síntesis de los resultados específicos de las consultas realizadas, como también, la descripción de algunos elementos definidos en el presente artículo, se ilustran en la tabla 14. De igual manera, en la tabla considerada, se listan por filas aquellos autores de los artículos evaluados, mientras que por columnas se identifican las variables analizadas. En consecuencia, cuando determinado autor (en filas), incluye en su contribución académica, cierta variable en particular (en columnas), se observa que en la tabla en mención, se registra el número "uno", en aquellas intersecciones correspondientes a las filas y a las columnas, relacionadas con el caso bajo estudio (autor vrs variable). Asimismo, en la siguiente tabla, se indica la clasificación de las variables de interés, según el componente de la sostenibilidad, al cual pertenezcan (social, ambiental o económico) y a su vez, se establece la fracción de artículos que participan en el tratamiento de cada variable.

De este modo, se concluye que en el proceso de programación de los sistemas Job Shop, el presente artículo corresponde al único desarrollo, por medio del cual se pretende analizar, las posibles relaciones del mencionado tipo de sistema de fabricación con el marco teórico de la salud en el trabajo. Adicionalmente, el artículo en cuestión, representa la única contribución que busca efectuar, la respectiva evaluación de las emisiones equivalentes de dióxido de carbono, generadas por el sistema de producción y por sus sistemas soporte. Con base en las argumentaciones anteriores, se plantea que el presente artículo, constituye la única propuesta, cuyo objetivo persigue la integración de los diversos componentes del enfoque sostenible (social, ambiental y económico), en aquel proceso asociado con la programación de los sistemas de 
manufactura bajo análisis. En este sentido, los resultados obtenidos en esta contribución, no pueden compararse con los resultados de menor alcance que se encuentran definidos, en los otros artículos analizados.

En la tabla 15, se muestra el total de variables integradas en cada artículo, como también se expone, la relación entre el número de variables de sostenibilidad analizadas en este artículo, respecto al número de variables de sostenibilidad, analizadas en los otros artículos. Al respecto, se determina que el método propuesto, supera a los otros métodos detectados, en cuanto al número de variables analizadas, así: $100 \%$, en $50 \%$ de los casos (tres de seis métodos evalúan 3 variables); $200 \%$ en $50 \%$ de los casos (tres de seis métodos evalúan 2 variables) y $140 \%$, en relación al número promedio de variables examinadas por los seis métodos en conjunto (6 variables en referencia a 2.5 variables).

Tabla 14: Síntesis de las consultas realizas para la programación sostenible en sistemas Job Shop.

\begin{tabular}{|c|c|c|c|c|c|c|c|c|}
\hline Enfoque & \multicolumn{3}{|c|}{ Social (salud en el trabajo) } & \multicolumn{2}{|c|}{ Ambiental } & \multicolumn{3}{|c|}{ Económico } \\
\hline Autores & $\begin{array}{l}\text { Intensidad } \\
\text { sonora }\end{array}$ & $\begin{array}{l}\text { Accidentes } \\
\text { laborales }\end{array}$ & $\begin{array}{c}\text { Peso } \\
\text { promedio } \\
\text { turno }\end{array}$ & $\begin{array}{l}\text { Emisión } \\
\text { integral de } \\
\mathrm{CO}_{2} \text { equiv }\end{array}$ & $\begin{array}{c}\text { Consumo } \\
\text { energía } \\
\text { Equipos }\end{array}$ & $\begin{array}{l}\text { Tiempo } \\
\text { proceso }\end{array}$ & $\begin{array}{c}\text { Costo } \\
\text { producción }\end{array}$ & Tardanza \\
\hline $\begin{array}{l}\text { Jiang et al } \\
(2014)\end{array}$ & & & & & 1 & 1 & 1 & \\
\hline $\begin{array}{l}\text { Liu et al } \\
(2014)\end{array}$ & & & & & 1 & & & 1 \\
\hline $\begin{array}{l}\text { Moon y } \\
\text { Park (2014) }\end{array}$ & & & & & 1 & & 1 & \\
\hline $\begin{array}{l}\text { Liu et al } \\
(2015)\end{array}$ & & & & & 1 & & 1 & 1 \\
\hline $\begin{array}{l}\text { May et al } \\
(2015)\end{array}$ & & & & & 1 & 1 & & \\
\hline $\begin{array}{l}\text { Raileanu et } \\
\text { al (2015) }\end{array}$ & & & & & 1 & 1 & 1 & \\
\hline $\begin{array}{l}\text { Este } \\
\text { artículo }\end{array}$ & 1 & 1 & 1 & 1 & & 1 & 1 & \\
\hline Total & 1 & 1 & 1 & 1 & 6 & 4 & 5 & 2 \\
\hline $\begin{array}{l}\text { Fracción } \\
\text { artículos } \\
\text { variable }\end{array}$ & $1 / 7$ & $1 / 7$ & $1 / 7$ & $1 / 7$ & $6 / 7$ & $4 / 7$ & $5 / 7$ & $2 / 7$ \\
\hline
\end{tabular}

Tabla 15: Número de variables de sostenibilidad por artículo.

\begin{tabular}{|l|c|c|c|c|c|c|c|c|}
\hline Autores de artículos & $\begin{array}{c}\text { Jiang } \\
\text { et al } \\
(2014)\end{array}$ & $\begin{array}{c}\text { Liu et } \\
\text { al } \\
(2014)\end{array}$ & $\begin{array}{c}\text { Moon y } \\
\text { Park } \\
(2014)\end{array}$ & $\begin{array}{c}\text { Liu et } \\
\text { al } \\
(2015)\end{array}$ & $\begin{array}{c}\text { May } \\
\text { et al } \\
(2015)\end{array}$ & $\begin{array}{c}\text { Raileanu } \\
\text { et al } \\
(2015)\end{array}$ & $\begin{array}{c}\text { Promedio } \\
\text { variables } \\
\text { por artículo }\end{array}$ & $\begin{array}{c}\text { Este } \\
\text { artículo }\end{array}$ \\
\hline Total variables por artículo & 3 & 2 & 2 & 3 & 2 & 3 & 2,5 & 6 \\
\hline $\begin{array}{l}\text { Comparación de No de } \\
\text { variables de este artículo } \\
\text { respecto a los otros artículos }\end{array}$ & $\begin{array}{c}100 \% \\
\text { por } \\
\text { arriba }\end{array}$ & $\begin{array}{c}200 \% \\
\text { por } \\
\text { arriba }\end{array}$ & $\begin{array}{c}200 \% \\
\text { por } \\
\text { arriba }\end{array}$ & $\begin{array}{c}100 \% \\
\text { por } \\
\text { arriba }\end{array}$ & $\begin{array}{c}200 \% \\
\text { por } \\
\text { arriba }\end{array}$ & $\begin{array}{c}100 \% \\
\text { por } \\
\text { arriba }\end{array}$ & $\begin{array}{c}140 \% \\
\text { por } \\
\text { arriba }\end{array}$ & \\
\hline
\end{tabular}

\section{Comparación del método propuesto con la forma de operación de la Organización evaluada.}

Según lo definido en el paso 11 de la metodología, se anota que en la actualidad, la organización objeto de estudio, analiza de forma detallada la obtención del tiempo de proceso. Para este efecto, se utiliza como regla de programación, aquella regla denominada: tiempo de operación más larga (LPT). Al aplicar la regla en mención, se obtienen 186 horas como tiempo de proceso. La implementación de este tiempo, genera sobre las otras variables evaluadas, el comportamiento mostrado enseguida: 1,94 semanas de tiempo de procesamiento (plan organizado en 16 horas al día y 6 días a la semana, de acuerdo con los lineamientos de la compañía), 0 puntos como penalidad para el costo de mano de obra directa, 4057,51 Kg de emisiones de dióxido de carbono equivalente, 1359 puntos como penalidad por accidentalidad laboral, 933,65 puntos como penalidad para el nivel de intensidad sonora y 1650,34 Kg manipulados en promedio por turno.

A partir de lo anterior, se establece que la compañía podría implementar aquellos individuos, cuyos tiempos de proceso sean cercanos o iguales, al menor tiempo de proceso (en horas). Al respecto, se explica que la última clase de tiempo, fue detectada por medio de la aplicación del método, aquí estructurado. A este tipo 
de tiempo, en el contexto de la empresa evaluada, le corresponde un valor de 160 horas. En la tabla 16, se presentan el plan actual implementado por tal organización (186 horas, en 16 horas al día y 6 días a la semana), acompañado de tres planes alternos (cercanos o iguales a 160 horas, en 16 horas al día y 6 días a la semana). Para el conjunto de los cuatro planes, se identifica su factor ponderado $\left(P\left(X_{j}\right)\right)$ y, a la vez para aquel plan alterno que presente la mayor valoración en este factor $\left(P\left(X_{j}\right)\right)$, se señala su comportamiento por variable (porcentualmente mayor, porcentualmente menor o igual valor), al ser comparado frente al plan de trabajo actual. De esta manera, el comité de dirección de la compañía, decide ejecutar aquel plan correspondiente a 160 horas de labor; definiendo a su vez, las medidas necesarias que permitan reducir el impacto negativo, respecto a la salud de las personas. En la figura 4, se aprecia el diagrama de Gantt para el individuo de menor tiempo de procesamiento.

Tabla 16: Plan actual y planes alternos.

\begin{tabular}{|l|c|c|c|c|c|c|c|}
\hline $\begin{array}{c}\text { Planes y } \\
\text { comparativos }\end{array}$ & $\begin{array}{c}\text { Tiempo } \\
\text { proceso } \\
\text { (semanas) }\end{array}$ & $\begin{array}{c}\text { Penalidad } \\
\text { costos } \\
\text { mano } \\
\text { obra } \\
\text { (puntos) }\end{array}$ & $\begin{array}{c}\text { Emisiones } \\
\text { equivalentes } \\
\text { dióxido de } \\
\text { carbono } \\
(\mathrm{Kg})\end{array}$ & $\begin{array}{c}\text { Penalidad } \\
\text { accidentes } \\
\text { laborales } \\
\text { (puntos) }\end{array}$ & $\begin{array}{c}\text { Penalidad } \\
\text { nivel de } \\
\text { intensidad } \\
\text { sonora } \\
\text { (puntos) }\end{array}$ & $\begin{array}{c}\text { Peso } \\
\text { promedio } \\
\text { turno } \\
(\mathrm{Kg})\end{array}$ & $\begin{array}{c}\text { Factor } \\
\text { ponderado } \\
\left(\mathrm{P}\left(\mathrm{X}_{\mathrm{j}}\right)\right)\end{array}$ \\
\hline $\begin{array}{l}\text { Plan base: } \\
186 \text { horas }\end{array}$ & 1.94 & 0 & 4057.12 & 1359 & 933.65 & 1650.34 & $50 \%$ \\
\hline Plan 160 horas & 1.66 & 0 & 4007.38 & 1360 & 950.807 & 1918.17 & $81.76 \%$ \\
\hline $\begin{array}{l}\text { Comparativos } \\
\text { plan 160 horas }\end{array}$ & $\begin{array}{c}\text { Disminuye } \\
14 \%\end{array}$ & $\begin{array}{c}\text { Igual } \\
\text { valor }\end{array}$ & $\begin{array}{c}\text { Disminuye } \\
1.2 \%\end{array}$ & $\begin{array}{c}\text { Incrementa } \\
0.22 \%\end{array}$ & $\begin{array}{c}\text { Incrementa } \\
1.8 \%\end{array}$ & $\begin{array}{c}\text { Incrementa } \\
16.27 \%\end{array}$ \\
\hline Plan 166 horas & 1.73 & 0 & 4019.46 & 1362 & 947.61 & 1849.18 & $73 \%$ \\
\hline Plan 171 horas & 1.78 & 0 & 4029.521 & 1376 & 945.11 & 1795.11 & $50 \%$ \\
\hline Mínimo & 1.66 & 0 & 4007.38 & 1359 & 933.65 & 1650.34 & \\
\hline Máximo & 1.94 & 0 & 4057.12 & 1376 & 950.807 & 1918.17 & \\
\hline
\end{tabular}

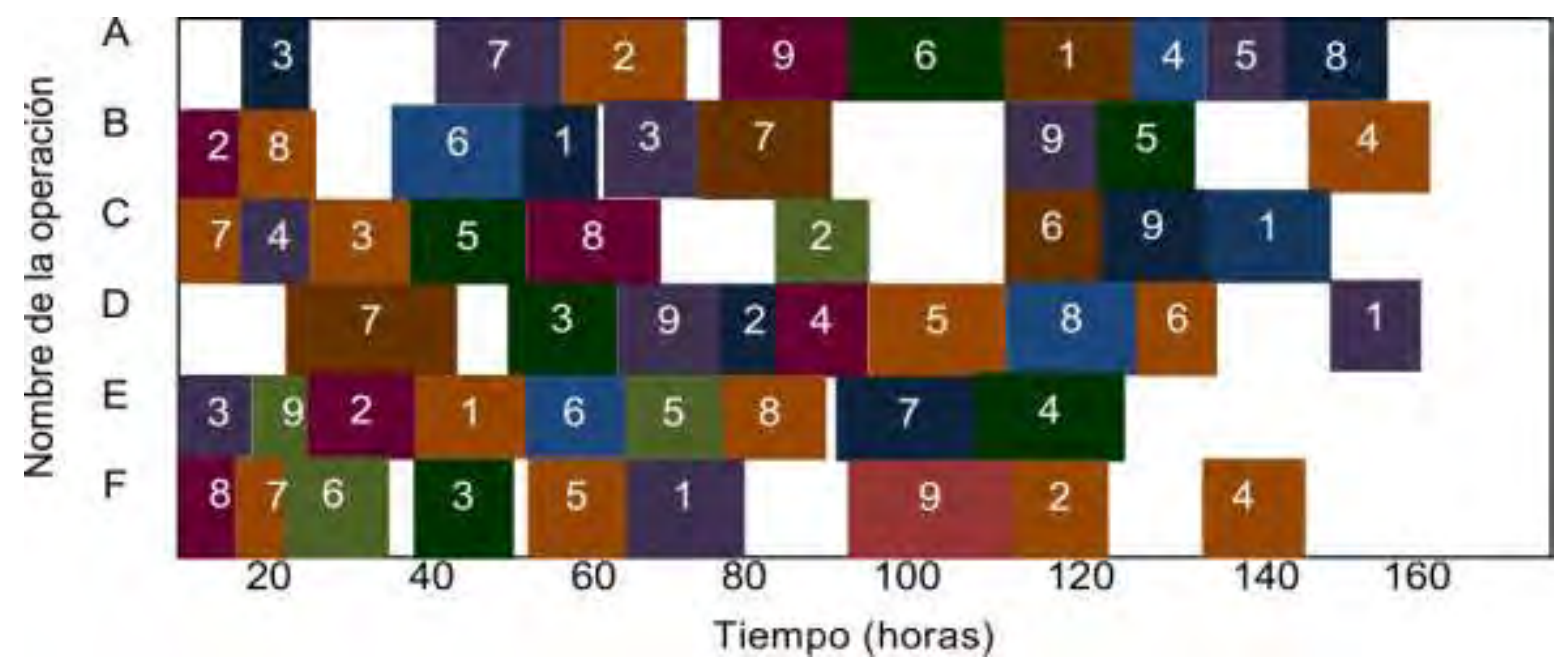

Fig. 4: Diagrama de Gantt para el individuo de menor tiempo de proceso. Tiempo de procesamiento: 160 horas equivalentes a 1,66 semanas; 0 puntos por mano de obra, 4007,38 Kg de $\mathrm{CO}_{2}, 1360$ puntos por accidentalidad; 950,81 puntos por intensidad sonora y $1918,17 \mathrm{Kg}$ de peso promedio

\section{Comparación del método propuesto con la aplicación de reglas de prioridad.}

Bajo las indicaciones del paso 12 de la metodología, se plantea en primera instancia la comparación del método propuesto, desde el comportamiento de la variable tiempo de proceso con la aplicación de algunas reglas de prioridad (LPT, SOT, FCFS, LCFS). Al realizar, el cotejo correspondiente, se detecta que el método diseñado muestra desempeño superior, al contrastarlo con los resultados establecidos, por medio de la aplicación de las reglas en cuestión. Este desempeño superior, se observa en el hecho de que el método estructurado, permite lograr mejores resultados en el comportamiento de la variable de interés, de acuerdo con lo expresado enseguida: menor en $14 \%$ respecto a LPT, menor en $20 \%$ respecto a SOT, menor en $30 \%$ respecto a LCFS y menor en $34 \%$ respecto a FCFS. En la tabla 17, se muestran los resultados particulares. 
Tabla 17: Método propuesto y reglas de programación (variable tiempo de proceso).

\begin{tabular}{|l|c|c|c|c|c|}
\hline $\begin{array}{c}\text { Método o } \\
\text { regla }\end{array}$ & $\begin{array}{c}\text { Tiempo proceso } \\
\text { (horas) }\end{array}$ & $\begin{array}{c}\text { Cotejo método } \\
\text { respecto a reglas }\end{array}$ & Regla & $\begin{array}{c}\text { Tiempo proceso } \\
\text { (horas) }\end{array}$ & $\begin{array}{c}\text { Cotejo método } \\
\text { respecto a reglas }\end{array}$ \\
\hline Método & 160 & - & LCFS & 227 & Menor en $30 \%$ \\
\hline LPT & 186 & Menor en $14 \%$ & FCFS & 241 & Menor en $34 \%$ \\
\hline SOT & 199 & Menor en $20 \%$ & & & \\
\hline
\end{tabular}

Asimismo, el comportamiento generado en las diversas variables evaluadas, debido a la implementación de los anteriores tiempos de proceso, al igual que la determinación del respectivo factor ponderado $\left(P\left(X_{j}\right)\right)$ para el conjunto de variables de interés, se observan en la tabla 18.

Tabla 18: Comportamiento de las variables relacionadas con los tiempos de proceso.

\begin{tabular}{|l|c|c|c|c|c|c|c|}
\hline $\begin{array}{c}\text { Método o } \\
\text { regla de } \\
\text { prioridad }\end{array}$ & $\begin{array}{c}\text { Tiempo } \\
\text { proceso } \\
\text { (semanas) }\end{array}$ & $\begin{array}{c}\text { Penalidad } \\
\text { costos } \\
\text { mano obra } \\
\text { (puntos) }\end{array}$ & $\begin{array}{c}\text { Emisiones } \\
\text { equivalentes } \\
\text { dióxido de } \\
\text { carbono }(\mathrm{Kg})\end{array}$ & $\begin{array}{c}\text { Penalidad } \\
\text { accidentes } \\
\text { laborales } \\
\text { (puntos) }\end{array}$ & $\begin{array}{c}\text { Penalidad } \\
\text { intensidad } \\
\text { sonora } \\
\text { (puntos) }\end{array}$ & $\begin{array}{c}\text { Peso } \\
\text { promedio } \\
\text { turno } \\
(\mathrm{Kg})\end{array}$ & $\begin{array}{c}\text { Factor } \\
\text { ponderado } \\
\left(\mathrm{P}\left(\mathrm{X}_{\mathrm{j}}\right)\right)\end{array}$ \\
\hline Propuesto & 1.66 & 0 & 4007.38 & 1360 & 950.81 & 1918.17 & 84.94 \\
\hline LPT & 1.94 & 0 & 4057.12 & 1359 & 933.65 & 1650.34 & 77.17 \\
\hline SOT & 2.07 & 0 & 4076.55 & 1382 & 925.72 & 1542.52 & 72.61 \\
\hline FCFS & 2.35 & 0 & 4212.13 & 1686 & 902.80 & 1358.56 & 35.82 \\
\hline LCFS & 2.50 & 0 & 4236.32 & 1690 & 891.24 & 1279.31 & 31.66 \\
\hline
\end{tabular}

En la figura 5, se aprecia el gráfico correspondiente al factor ponderado que se estimó en la tabla 18. En este caso, se aprecia que el método estructurado supera el desempeño de las reglas de programación, debido a que el primero, presenta mayor valor de "factor ponderado", así: $10 \%$ respecto a LPT, $17 \%$ respecto a SOT, $137 \%$ respecto a FCFS y $168 \%$ respecto a LCFS. Además, se afirma que la dirección de la compañía frente a la implementación de un plan laboral de 160 horas, requiere tomar medidas de mitigación pertinentes.

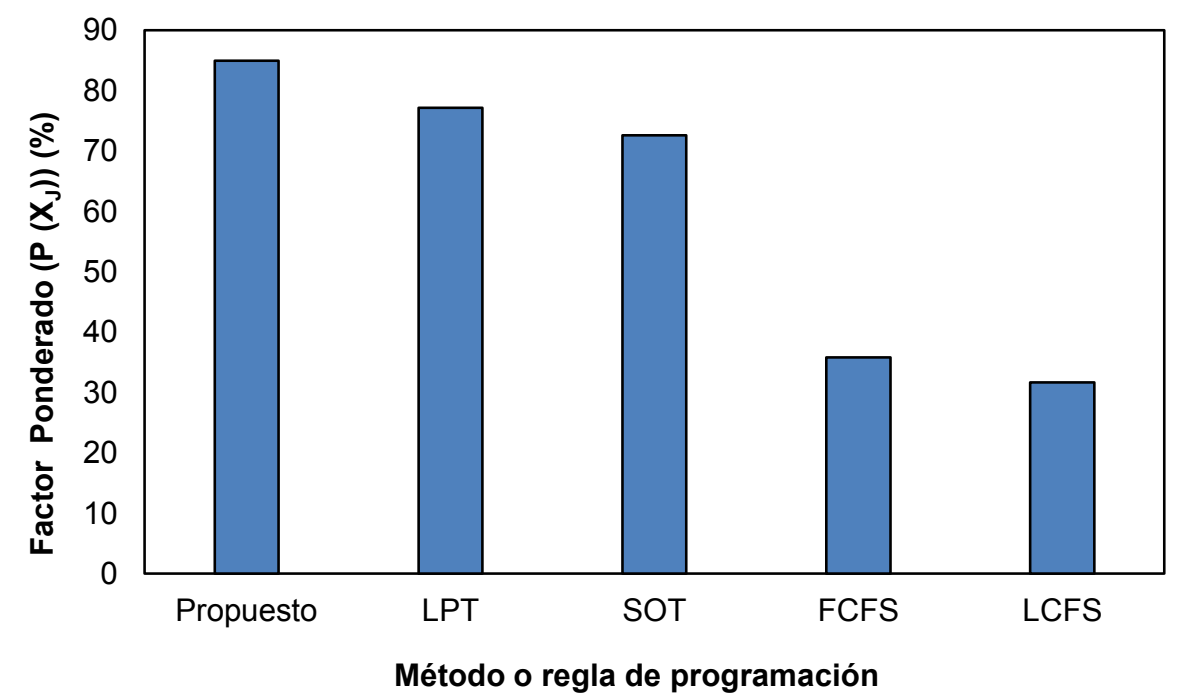

Fig. 5: Factor ponderado $P\left(X_{j}\right)$ para método o regla de programación.

\section{Comparación del método propuesto con la aplicación del método "cuello de botella móvil".}

En referencia al paso 13 de la metodología y a partir de la información proveniente de la tabla 3 , se procede a registrar en la tabla 19, aquellos valores correspondientes a los siguientes parámetros: carga por centro de trabajo (ordenada de forma descendente); comportamiento porcentual de la carga por centro (carga por centro en relación a la carga total) y finalmente, nivel porcentual de carga acumulada en cada centro. De esta manera, se determinan aquellas operaciones bajo las cuales se acumula hasta el $50 \%$ de la carga 
total de fabricación. En este sentido, se expresa que las operaciones en cuestión, constituyen los cuellos de botella móviles. Con base en lo anterior, se argumenta que los centros de trabajo restricción para la situación particular analizada en el presente artículo, están representados por las operaciones E, A y C.

Tabla 19: Análisis de carga en los centros de trabajo.

\begin{tabular}{|l|c|c|c|c|c|c|c|}
\hline Centro de trabajo & Centro E & Centro A & Centro C & Centro B & Centro F & Centro D & Total carga \\
\hline Carga por centro (horas) & 123 & 121 & 119 & 117 & 115 & 113 & 708 \\
\hline Carga porcentual centro (\%) & 17.37 & 17.09 & 16.81 & 16.53 & 16.24 & 15.96 & 100 \\
\hline Carga acumulada centro (\%) & 17.37 & 34.46 & 51.27 & 67.81 & 84.04 & 100 & \\
\hline
\end{tabular}

Con base en lo ilustrado, se señala que la existencia de tres operaciones restricción, en rutas de fabricación variables, genera como requerimiento la necesidad de identificar e implementar aquella secuencia de programación de los pedidos, por medio de la cual se obtenga el menor tiempo de manufactura. Para este efecto, se aprecia en la tabla 20, la determinación del "factor restricción" (Fr), cuyo valor se estima relacionando la carga de fabricación de los pedidos en las operaciones "cuello de botella", respecto a la carga total de trabajo (ver tabla 19). Complementariamente, se menciona que las operaciones restricción presentan asignaciones de tiempo diferentes, de acuerdo con las especificaciones técnicas de cada pedido. De este modo, un primer tipo de "orden de producción" demandará mayor asignación de tiempo en las operaciones "cuello de botella", mientras que un segundo tipo, usará en menor grado tal categoría de recursos. A partir de lo descrito, se anota que la medición correspondiente a la variable "factor restricción", arroja comportamientos diversos para cada clase de "orden de trabajo". De manera consecuente, al efectuar la comparación entre aquellos valores de "factor restricción" establecidos por cada tipo de "orden de producción", se detecta a nivel general que aquel valor definido para el primer tipo, supera a aquel valor calculado para el segundo tipo. De la tabla 20 se deduce que el pedido 8 utiliza en mayor grado las operaciones restricción y a su vez, se concluye que el pedido 5 utiliza en menor grado los recursos cuello de botella.

Tabla 20: Factor restricción por pedido en operaciones restricción (Fr).

\begin{tabular}{|l|c|c|c|c|c|c|c|c|c|}
\hline NN$^{\circ}$ Pedido & 1 & 2 & 3 & 4 & 5 & 6 & 7 & 8 & 9 \\
\hline Tiempo operación en centro E & 12 & 12 & 16 & 17 & 13 & 11 & 16 & 15 & 11 \\
\hline Tiempo operación en centro A & 14 & 15 & 12 & 10 & 7 & 17 & 14 & 14 & 18 \\
\hline Tiempo operación en centro C & 15 & 12 & 11 & 14 & 12 & 10 & 14 & 17 & 14 \\
\hline Total tiempo en operaciones críticas & 41 & 39 & 39 & 41 & 32 & 38 & 44 & 46 & 43 \\
\hline Factor restricción (Fr) (\%) & 5.79 & 5.51 & 5.51 & 5.79 & 4.52 & 5.37 & 6.21 & 6.49 & 6.07 \\
\hline
\end{tabular}

En la tabla 21, se observan las secuencias detectadas con el fin de obtener los programas específicos de producción. A su vez, se enuncia que las posiciones asignadas a cada pedido en las mencionadas secuencias, se establecieron ordenando de manera descendente, aquellos valores calculados en la tabla 20 para la variable denominada "factor restricción" ( $\mathrm{Fr}$ ). Al respecto, se indica que los siguientes pares de pedidos: "pedido № 1" comparado con "pedido № 4" y asimismo, "pedido № 2" comparado con "pedido № 3", presentan el mismo valor, en cuanto al comportamiento de la variable "Fr". De esta forma, se aprecian en la tabla 21 las cuatro secuencias de producción a ser analizadas.

Tabla 21: Secuencias de producción a ser analizadas (método cuello de botella móvil).

\begin{tabular}{|l|c|c|c|c|c|c|c|c|c|c|c|}
\hline $\begin{array}{l}\mathrm{N}^{\circ} \text { posición pedido en el } \\
\text { programa de producción. }\end{array}$ & 1 & 2 & 3 & 4 & 5 & 6 & 7 & 8 & 9 & $\begin{array}{c}\text { Tiempo proceso } \\
\text { (horas) }\end{array}$ & $\begin{array}{c}\text { Cotejo método propuesto } \\
\text { respecto a secuencias }\end{array}$ \\
\hline $\mathrm{N}^{\circ}$ pedido en $1^{a}$ secuencia. & 8 & 7 & 9 & 1 & 4 & 2 & 3 & 6 & 5 & 165 & Menor en $3.03 \%$. \\
\hline $\mathrm{N}^{\circ}$ pedido en $2^{a}$ secuencia. & 8 & 7 & 9 & 1 & 4 & 3 & 2 & 6 & 5 & 165 & Menor en $3.03 \%$. \\
\hline $\mathrm{N}^{\circ}$ pedido en $3^{a}$ secuencia. & 8 & 7 & 9 & 4 & 1 & 2 & 3 & 6 & 5 & 166 & Menor en $3.61 \%$. \\
\hline $\mathrm{N}^{\circ}$ pedido en $4^{\text {a }}$ secuencia. & 8 & 7 & 9 & 4 & 1 & 3 & 2 & 6 & 5 & 167 & Menor en $4.19 \%$. \\
\hline
\end{tabular}

En la tabla 22, se ilustran los resultados definidos a partir de la aplicación de ambos métodos (propuesto y cuello de botella móvil). De este modo, se establece que el método propuesto supera el desempeño correspondiente al método de cuello de botella móvil, en cuanto al comportamiento de la variable genérica "factor ponderado" $\left(P\left(X_{j}\right)\right)$, así: $2,2 \%$ en relación a los resultados arrojados por la primera secuencia; $2,1 \%$ en relación a los resultados arrojados por la segunda secuencia; $2,5 \%$ en relación a los resultados arrojados por la tercera secuencia y 3,1 \% en relación a los resultados arrojados por la cuarta secuencia. 
Tabla 22: Comportamiento de variables (método propuesto y secuencias del método de cuello de botella móvil).

\begin{tabular}{|l|c|c|c|c|c|c|c|}
\hline $\begin{array}{c}\text { Método o } \\
\text { secuencia } \\
\text { en cuello de } \\
\text { botella móvil }\end{array}$ & $\begin{array}{c}\text { Tiempo } \\
\text { proceso } \\
\text { (sem) }\end{array}$ & $\begin{array}{c}\text { Penalidad } \\
\text { costos } \\
\text { mano obra } \\
\text { (puntos) }\end{array}$ & $\begin{array}{c}\text { Emisiones } \\
\text { equivalentes } \\
\text { dióxido de } \\
\text { carbono }(\mathrm{Kg})\end{array}$ & $\begin{array}{c}\text { Penalidad } \\
\text { accidentes } \\
\text { laborales } \\
\text { (puntos) }\end{array}$ & $\begin{array}{c}\text { Penalidad } \\
\text { intensidad } \\
\text { sonora } \\
\text { (puntos) }\end{array}$ & $\begin{array}{c}\text { Peso } \\
\text { promedio } \\
\text { turno } \\
(\mathrm{Kg})\end{array}$ & $\begin{array}{c}\text { Factor } \\
\text { ponderado } \\
\left(\mathrm{P}\left(\mathrm{X}_{\mathrm{j}}\right)\right)\end{array}$ \\
\hline Propuesto & 1.66 & 0 & 4007.38 & 1360 & 950.81 & 1918.17 & 84.94 \\
\hline $1^{\mathrm{a}}$ secuencia & 1.72 & 0 & 4017.45 & 1362 & 948.04 & 1860.82 & 83.11 \\
\hline $2^{\mathrm{a}}$ secuencia & 1.72 & 0 & 4017.83 & 1360 & 948.04 & 1860.82 & 83.17 \\
\hline $3^{\text {a } \text { secuencia }}$ & 1.73 & 0 & 4019.42 & 1361 & 947.60 & 1849.61 & 82.85 \\
\hline $4^{\text {a }}$ secuencia & 1.74 & 0 & 4021.47 & 1362 & 947.07 & 1838.51 & 82.42 \\
\hline
\end{tabular}

Evaluación de la confiablidad del algoritmo genético a nivel de experimentación.

Según lo indicado en el paso № 14 de la metodología, se procedió a ejecutar el programa durante 30 oportunidades. De este modo, se definieron 30 poblaciones de respuesta. Más adelante, se determinaron los valores promedio de las variables de interés (tiempo de procesamiento en semanas, costos de mano de obra directa, emisiones equivalentes de dióxido de carbono, accidentalidad laboral, nivel de intensidad sonora y peso promedio manipulado por turno), en cada población de respuesta. Una vez, calculados los valores promedio, se procedió a realizar el respectivo análisis de varianza, utilizando con este propósito: 2 tratamientos, 15 repeticiones y un nivel de confianza de $95 \%$. En la tabla 23, se aprecia la síntesis de los datos base de evaluación para aquellos parámetros objeto de estudio, acompañados de su respectivo anova.

Tabla 23: Análisis de varianza para variables de sostenibilidad.

\begin{tabular}{|l|c|c|c|c|c|c|}
\hline & $\begin{array}{c}\text { Promedio } \\
\text { tiempo } \\
\text { proceso }\end{array}$ & $\begin{array}{c}\text { Promedio } \\
\text { costo mano } \\
\text { de obra } \\
\text { directa }\end{array}$ & $\begin{array}{c}\text { Promedio } \\
\text { emisiones } \\
\text { equivalentes } \\
\text { dióxido carbono }\end{array}$ & $\begin{array}{c}\text { Promedio } \\
\text { accidentes } \\
\text { laborales }\end{array}$ & $\begin{array}{c}\text { Promedio } \\
\text { nivel } \\
\text { intensidad } \\
\text { sonora }\end{array}$ & $\begin{array}{c}\text { Promedio } \\
\text { peso } \\
\text { manipulado } \\
\text { turno }\end{array}$ \\
\hline $\begin{array}{l}\text { Valor promedio repetición } \\
\text { No 1 - Tratamiento uno }\end{array}$ & 2.01 & 1334.40 & 4137.67 & 1500.13 & 918.93 & 1486.24 \\
\hline $\begin{array}{l}\text { Valor promedio repetición } \\
\text { No 15 - Tratamiento uno }\end{array}$ & 1.95 & 1342.80 & 4136.30 & 1515.03 & 918.72 & 1485.18 \\
\hline $\begin{array}{l}\text { Valor promedio repetición } \\
\text { No 1 - Tratamiento dos }\end{array}$ & 2.08 & 1341.27 & 4138.67 & 1501.50 & 917.92 & 1484.24 \\
\hline $\begin{array}{l}\text { Valor promedio repetición } \\
\text { No 15 - Tratamiento dos }\end{array}$ & 2.06 & 1338.29 & 4137.35 & 1503.15 & 918.22 & 1486.18 \\
\hline $\begin{array}{l}\text { Suma cuadrados } \\
\text { tratamientos }\end{array}$ & 0.00196 & 5689.49 & 9.69 & 12.055 & 0.43 & 1.24 \\
\hline $\begin{array}{l}\text { Suma cuadrados error } \\
\text { experimental }\end{array}$ & 0.004764 & 164089.09 & 100.43 & 2806.45 & 5.73 & 19.06 \\
\hline $\begin{array}{l}\text { GL tratamientos - error } \\
\text { experimental - total }\end{array}$ & $1-28-29$ & $1-28-29$ & $1-28-29$ & $1-28-29$ & $1-28-29$ & $1-28-29$ \\
\hline Valor prueba F calculado & 0.41 & 0.971 & 2.71 & 0.12 & 2.088 & 1.82 \\
\hline Probabilidad & 0.53 & 0.332 & 0.1114 & 0.73 & 0.1595 & 0.182 \\
\hline Valor prueba F crítico & 4.19 & 4.19 & 4.19 & 4.19 & 4.19 & 4.19 \\
\hline
\end{tabular}

A partir de lo anterior, se observa que el valor de la distribución $\mathrm{F}$ establecida con el siguiente nivel alfa y grados de libertad: $(0,05,1,28)$, es superior al valor de las distribuciones $\mathrm{F}$ calculadas sobre el mismo punto. Asimismo, se determina que el valor $\mathrm{P}$ supera en todos los casos el nivel alfa de $5 \%$. Con base en lo afirmado, se concluye que los datos arrojados por el método propuesto son estadísticamente iguales, en lo que respecta al comportamiento específico de cada una de las variables de interés.

En la revisión de la literatura evaluada (Davarzani \& Akbarzadeh-T, 2014; Nicoara, 2012; Nagamani et al., 2013; Rudy \& Zelasny, 2015; Xiong et al., 2012; Xue et al., 2014; Yang y Gu, 2014; Yuan \& Xu, 2013) y sus antecedentes, se expone que los procesos de investigación de los sistemas Job Shop, se han consolidado durante los últimos años, en ciertos aspectos relacionados, con el desarrollo de algoritmos conceptualmente 
novedosos, en el área de la metaheurística. De este modo, se pretende lograr dos objetivos básicos: mejorar el desempeño de variables tradicionales de interés (tiempo de proceso, carga de trabajo en operación crítica, total de carga de trabajo) o reducir los tiempos de cómputo, correspondientes a aquellos programas informáticos, bajo los cuales se representan los algoritmos en consideración.

Igualmente, se establece que en la actualidad, la programación de los sistemas Job Shop, al tomar referencia en un enfoque sostenible, presenta en términos generales, el siguiente estado de desarrollo: evaluación amplia en eficiencia operacional, evaluación reducida en el área de la salud ocupacional y evaluación parcial en el área de gestión ambiental. Con relación al último aspecto, se anota que desde la perspectiva ambiental, las 6 contribuciones académicas detectadas, realizan énfasis en la aplicación de procesos de sub-optimización, asociados con la minimización del consumo de energía; sin embargo, no se identifican avances de investigación, en otros aspectos relevantes (medición de residuos sólidos y de aguas residuales).

En cuanto al método de programación actual del sistema de operaciones de la empresa objeto de estudio, se menciona que tal compañía, identifica el valor correspondiente al tiempo de proceso, usando la regla LPT. De forma consecuente, se enuncia que la aplicación de esta regla, permite definir un valor de 186 horas, como valor de tiempo de manufactura. Complementariamente, se expresa que después de ejecutar el método propuesto, se detectó como valor de tiempo de fabricación, un valor que asciende a 160 horas. Es así como, respecto a los tiempos señalados, se indica que el hecho de migrar de un plan de programación elaborado para 186 horas, hacia un plan de fabricación estructurado para 160 horas, tendría los siguientes efectos, sobre las variables de interés: reducción de $14 \%$ en tiempo de proceso (semanas), reducción de $1,2 \%$ en emisiones de dióxido de carbono equivalentes, incremento de $0,12 \%$ en accidentalidad laboral, incremento de 1,8\% en nivel de intensidad sonora e incremento de $16,27 \%$ en peso promedio manipulado por turno.

En cuanto al método metaheurístico propuesto, se determina su capacidad de obtener respuestas diversas, debido a que se integran de manera pertinente, los siguientes conceptos: carga de trabajo, búsqueda local y algoritmos genéticos. De igual forma, se establece que el método en cuestión, supera en desempeño tanto al método de cuello de botella móvil como a la aplicación de reglas de prioridad, en relación al comportamiento de la variable denominada: "factor ponderado", así: 2,5\% en promedio, respecto al método de cuello de botella móvil y $83 \%$ en promedio, respecto a las reglas de prioridad. Complementariamente, se anota que el método estructurado, presenta características de confiabilidad, lo cual se evidencia en la estabilidad estadística de las respuestas logradas al ejecutar el programa de cómputo (en el caso de los 6 parámetros analizados, el nivel $\mathrm{F}$ calculado es inferior al valor $\mathrm{F}$ crítico y a su vez, el valor $\mathrm{P}$ es superior al nivel alfa de $5 \%$ ).

\section{CONCLUSIONES}

Con base en los resultados encontrados y su discusión se concluye que:

1) El método diseñado puede ser replicado por las personas interesadas en el tema, puesto que se definen sus pasos con claridad y, a su vez se caracteriza de manera detallada, la información de entrada requerida. 2) El método propuesto, debido a su capacidad de superar al método de cuello de botella móvil, en cuanto al comportamiento del factor ponderado $\left(P\left(X_{j}\right)\right)$, muestra su potencialidad para ser comparado con metaheurísticas multiobjetivo.

3) En la literatura de corriente principal, el enfoque actual de la sostenibilidad para la programación de los sistemas Job Shop, no ha integrado de manera significativa, aquellos elementos por medio de los cuales, se estructure su componente social. Por tanto, se determina la existencia de variadas oportunidades académicas de desarrollo, en lo referente al componente en mención.

4) Desde el enfoque de la sostenibilidad a nivel ambiental, en la programación de los sistemas Job Shop, se ha priorizado el análisis del consumo de energía. De esta forma, se identifica una amplia diversidad de oportunidades, para construir las relaciones existentes entre el tiempo de proceso con ciertos fenómenos que afectan el medio ambiente: contaminación por emisiones gaseosas diferentes a emisiones de dióxido de carbono, contaminación por disposición de aceites, entre otras

5) Aunque el componente económico de la sostenibilidad, en la programación de los sistemas Job Shop, se encuentra extensivamente explorado, sus relaciones con los componentes social y ambiental, todavía deben investigarse y consolidarse a nivel teórico y práctico, de forma coherente.

6) En el marco de los individuos no dominados, la compañía cuenta con una gama representativa de opciones de solución, las cuales deben analizarse de acuerdo con cada contexto, procediendo a implementar en lo posible, aquellos individuos que logren un mejor equilibrio entre los componentes social, ambiental y económico de la sostenibilidad. 


\section{AGRADECIMIENTOS}

Se agradece de forma especial a la Universidad Nacional de Colombia -sede Manizales-, por su apoyo en la ejecución del proyecto que actualmente adelantan los autores del artículo, bajo las normas del programa de Doctorado en ingeniería -Industria y Organizaciones- y, cuyos resultados parciales, se ilustran en este trabajo.

\section{REFERENCIAS}

Bečić, E. Mulej, E. M. y Švarc, J., Measuring Social Progress by Sustainable Development Indicators: Cases of Croatia and Slovenia, doi:10.1016/j.sbspro.2012.03.311, Procedia - Social and Behavioral Sciences, 37, 458-465 (2012)

Castrillón, O. Sarache, W. y Giraldo, J., Aplicación de un algoritmo evolutivo en la solución de problemas Job Shop-Open Shop, doi:10.4067/S0718-07642011000100011, Inf. Tecnol. 22(1), 83-92 (2011)

Davarzani, Z. y Akbarzadeh, T., A novel Quantum Inmmune Algorihm for Multibobjective Flexible Job Shop Scheduling, doi:10.1142/S0218213014500067, International Journal of Artificial Intelligence and Applications for Smart Devices, 2(1), 57-72 (2014)

Fang, Y. y Yue, Y., An improved genetic algorithm to the job shop scheduling problem, http://jocpr.com/vol7iss4-2015/JCPR-2015-7-4-322-325.pdf, ISSN: 0975-7384, Journal of Chemical and Pharmaceutical Research, 7(4), 322-325 (2015)

Ghiasi, H. Damiano, P. y Lessard, L., A Non-dominated sorting hybrid algorithm for multi-objective optimization of engineering problems, doi:10.1080/03052151003739598, Engineering Optimization, 43(1), 39-59 (2012)

Goldratt, E. y Cox, J., La Meta, $3^{a}$ edición, 316-344. Díaz de Santos, Buenos Aires, Argentina (2005)

Huang, X. Zhao, X. y Ma, X., An improved genetic algorithm for job-shop scheduling problem with process sequence flexibility, doi:10.4028/www.scientific.net/AMR.97-101.2449, International Journal of Simulation Modelling, 13(4), 510-522 (2014)

Jiang, Z. Zuo, L. y E, M., Study on Multi-objective Flexible Job - Shop Scheduling Problem considering Energy Consumption, doi:10.3926/jiem.1075, J. Ind. Engineering and Management, 7(3), 568-604 (2014)

Karimi-Nasab, M. Modarres, M. y Seyedhoseini, S. M., A self-adaptive PSO for joint lot sizing and job shop scheduling with compressible process times, doi:10.1016/j.asoc.2014.10.015, Applied Soft Computing, 27 , 137-147 (2015)

Liu, Y. Dong, H. Lohse, N. y Petrovic, S., Reducing environmental impact of production during a Rolling Blackout policy - A multi-objective schedule optimisation approach, doi:10.1016/j.jclepro.2015.04.038, Journal of Cleaner Production, 102, 418-427 (2015)

Liu, Y. Dong, H. Lohse, N. Petrovic, S. y Gindy, N., An investigation into minimising total energy consumption and total weighted tardiness in job shops, doi:10.1016/j.jclepro.2013.07.060, Journal of Cleaner Production, $65,87-96(2014)$

López, J. Giraldo, J. y Arango, J., Reducción del tiempo de terminación en la programación de la producción de una línea de flujo híbrida flexible (HFS), doi:10.4067/S0718-07642015000300019, Información Tecnológica, 26(3), 157-172 (2015)

May, G. Stahl, B. Taisch, M. y Prabhu, V., Multi-objective genetic algorithm for energy-efficient job shop scheduling, doi:10.1080/00207543.2015.1005248, Int. j. Production Research, 53(23), 7071-7089 (2015)

Mencía, R. Sierra, M. Mencía, C. y Varela, R., A genetic algorithm for job-shop scheduling with operators enhanced by weak Lamarckian evolution and search space narrowing, doi:10.1007/s11047-013-9373-x, Natural Computing, 13(2), 179-192 (2014)

Moon, J. y Park, J., Smart production scheduling with time-dependent and machine-dependent electricity cost by considering distributed energy resources and energy storage, doi:10.1080/00207543.2013.860251, International Journal of Production Research, 52(13), 3922-3939 (2014) 
Nagamani, M. Chandrasekaran, E. y Saravanan, D., Multiobjective Evolutionary Algorithm For Flexible Job Shop Scheduling Problem Minimization of Makespan, Maximum Workload and Total Workload of Machines, http://www.academia.edu/3311788, ISSN: 2249-6955, International Journal of Mathematics and Computer, 3(2), 79-86 (2013)

Nicoara, E., Multi-objective Flexible Job Shop Scheduling Optimization Models, http://www.upg-bulletinse.ro/archive/2012-3/10.Nicoara.pdf, ISSN: 2284-8584, Economic Insights - Trends and challenges, 49 (2), 79-86 (2012)

Pagell, M. y Shevchenko, A., Why research in sustainable supply chain management should have no future, doi:10.1111/jscm.12037, Development of Truly Sustainable Supply Chains, 50(1), 44-55 (2014)

Raileanu, S. Anton, F. y Latan, A., Optimizing Power Consumption in Robotized Job-Shop Manufacturing, doi:10.1007/978-3-319-15159-5_2, Service Orientation in Holonic and Multi-agent Manufacturing, 594, 13-27 (2015)

Rudy, J. y Zelasny, D., Solving multi-objective Job Shop problem using nature - based algorithms: new Pareto approximation features, doi:10.11121/ijocta.01.2015.00232, An International Journal of Optimization and Control: Theories \& Applications, 5(1), 1-11 (2015)

Ruiz, S. Castrillón, O. y Sarache, W., Una metodología multiobjetivo para optimizar un ambiente Job Shop, doi:10.4067/S0718-07642012000100005, Información Tecnológica, 23 (1), 35-46 (2012)

Shen, X. Zhang, M. y Fu, J., Multiobjective Dynamic Job Shop Scheduling a Survey and Propects, http://www.ijicic.org/ijicic-14-01022.pdf, ISSN: 1349-4198, International Journal of Innovative, 10(6), 21132126 (2014)

Shen, X. y Yao, X., Mathematical modeling and multi-objective evolutionary algorithms applied to dynamic flexible job shop scheduling problems, doi:10.1016/j.ins.2014.11.036, Information Sciences, 298, 198-224 (2015)

Xiong, J. Tan, X. Yang, W. Xing, N. y Chen, W., A Hybrid Multiobjective Evolutionary Approach for Flexible Job-Shop Scheduling Problems, doi: 10.1155/2012/478981, Mathematical problems in engineering, Volumen 2012, ID 478981, 27 p (2012)

Xue, H. Zhang, P. Wei, Z. y Yang, L., An improved Immune Algorithm for Multiobjective Flexible Job-Shop Scheduling, doi:10.4304/jnw.9.10.2843-2850, Journal of Networks, 9(10), 2843-2850 (2014)

Yang, Y. y Gu, X., Cultural-Based Genetic Tabu Algorithm for Multiobjective Job Shop Scheduling, doi:10.1155/2014/230719, Mathematical Problems in Engineering, Volumen 2014, Article ID 230719, 14p (2014)

Yuan, Y. y Xu, H., A memetic algorithm for the multi-objective flexible job shop scheduling problem, doi:10.1145/2463372.2463431, Proceedings of the 15th annual conference on Genetic and evolutionary computation Gecco'13, Amsterdam, The Netherlands, 559-566 (2013) 